\title{
A Toy Model of the Instability in the Equatorially Trapped Convectively Coupled Waves on the Equatorial Beta Plane
}

\section{Citation}

Andersen, Joseph A. and Zhiming M. Kuang. 2008. A toy model of the instability in the equatorially trapped convectively coupled waves on the equatorial beta plane. Journal of Atmospheric Sciences 65(12): 3736-3757.

\section{Published Version}

http://dx.doi.org/10.1175/2008JAS2776.1

\section{Permanent link}

http://nrs.harvard.edu/urn-3:HUL.InstRepos:3201851

\section{Terms of Use}

This article was downloaded from Harvard University's DASH repository, and is made available under the terms and conditions applicable to Open Access Policy Articles, as set forth at http:// nrs.harvard.edu/urn-3:HUL.InstRepos:dash.current.terms-of-use\#OAP

\section{Share Your Story}

The Harvard community has made this article openly available.

Please share how this access benefits you. Submit a story.

\section{Accessibility}




\section{ABSTRACT}

\section{A toy model of the instability in the}

\section{equatorially trapped convectively coupled}

\section{waves on the equatorial beta plane}

Joseph Allan Andersen*

Department of Physics, Harvard University,

Cambridge, Massachusetts

ZHIMING KUANG

Department of Earth and Planetary Science and

School of Engineering and Applied Sciences,

\section{Harvard University,}

Cambridge, Massachusetts

\footnotetext{
${ }^{*}$ Corresponding author address: Joseph Allan Andersen, Department of Physics, Harvard University,
} Cambridge, MA 02138

E-mail: jaanders@fas.harvard.edı
The equatorial atmospheric variability shows a spectrum of significant peaks in the wave number-frequency domain. These peaks have been identified with the equatorially trapped wave modes of rotating shallow water wave theory. This paper addresses the observation that the various wave types (e.g. Kelvin, Rossby, etc.) and wave numbers show differing signal strength relative to a red background. It is hypothesized that this may be due to variations in the linear stability of the atmosphere to the various wave types depending both upon the specific wave type and the wavenumber. A simple model of the convectively coupled waves on the equatorial beta plane is constructed to identify processes that contribute to this dependence. The linear instability spectrum of the resulting coupled system is evaluated by eigenvalue analysis. This analysis shows unstable waves with phase speeds, growth rates, and structures (vertical and horizontal) that are broadly consistent with the results from observations. The linear system shows peak unstable Kelvin waves around zonal wavenumber seven with peak growth rates of $\sim 0.08 /$ day (e-folding time of $\sim 13$ days). The system also shows unstable Mixed Rossby-Gravity (MRG) and Inertio-Gravity waves with significant growth in the zonal wavenumber range from negative (westward phase speed) fifteen to positive (eastward phase speed) ten. The peak MRG/n=0 EIG growth rate is around one third that of the Kelvin wave and occurs at zonal wavenumber three. The Rossby waves in this system are stable, and the MaddenJulian Oscillation is not observed. Within this model, it is shown that besides the effect of the Inter-Tropical Convergence Zone configuration, the differing in- 
stabilities of the different wave modes are also related to their different efficiency in converting input energy into divergent flow. This energy conversion efficiency difference is suggested as an additional factor that helps to shape the observed wave spectrum.

\section{Introduction}

If the satellite record of outgoing long-wave radiation (Liebman and Smith 1996), OLR, - a good proxy for deep tropical convection (see, for example Arkin and Andanuy (1989)) within 15 degrees of the equator is analyzed in zonal wavenumber-frequency space (Wheeler and Kiladis 1999), it shows a number of statistically significant peaks (figure 1). These propagating disturbances form a large part of the tropical synoptic scale variation, organizing individual convective elements (typically 100km across, surviving for a few hours) on large spatial (thousands of kilometers) and temporal (days) scales (e.g. Chang (1970), Nakazawa (1988)). The wave activity peaks have been identified with the equatorially trapped waves of shallow water theory (see, for example: Wheeler and Kiladis (1999), Yang et al. (2007)). Many of the properties of these waves are well described by rotating shallow water wave theories (Matsuno 1966). Here we attempt to address the observation that the various wave types (e.g. Kelvin, Rossby, etc) and wave-numbers show differing amplitudes. It is hypothesized that this is due, at least in part, to the (linear) stability of the atmosphere to perturbations of the various wave types depending upon the specific wave type and the wave number in question.

We explore this using the simple model of the convectively coupled waves using the first two baroclinic vertical modes, mid-tropospheric moisture, and the sub-cloud layer developed in Kuang (2008b), extended to the equatorial beta plane. The model of Kuang (2008b) is part of a continuing effort in this field to construct models of minimal complexity to identify the basic instability mechanisms of these waves. There is a long history of such simple models, going back at least as far as 1970 (e.g. Havashi, Y. A theory of large-scale equatorial 
waves generated by condensation heat and accelerating the zonal wind. J. Meteorol. Soc. Japan., 48, 140-160, Lindzen (1974), Neelin et al. (1987), Wang (1988), Mapes (2000), Majda and Shefter (2001), Khoudier and Majda (2006a), Raymond and Fuchs, 2007). The early models emphasized a first baroclinic structure that is of one sign over the full depth of the troposphere (e.g. Wang (1988),Neelin et al. (1987),Emanuel87). The importance of the second baroclinic component was recognized and included in the model of Mapes (2000) and an instability mechanism involving the second baroclinic temperature anomaly and deep convective and stratiform heating was identified Mapes (2000). More recently, Khoudier and Majda (2006a) included the interaction between free tropospheric moisture and deep convective and congestus heating in their model and found that moisture plays a major role in destabilizing the system. This is an important finding. However, the actual instability mechanism was not clearly identified. Khoudier and Majda (2006a) described the process of a dry episode causing more congestus heating, which moistens and preconditions the free troposphere, leading to deep convection, which then returns the free troposphere to a dry condition. This description, while correct, does not reveal how the initial perturbation gets amplified, thus falls short of an instability mechanism. This motivated the study of Kuang (2008b), which continued the effort by Khoudier and Majda (2006a) but seeked to develop conceptually simple treatments of convection and to identify the basic instability mechanisms.

In Kuang (2008b), the convective parameterization is based upon the quasi-equilibrium concept - that convection responds quickly to the changes in the large-scale flow, so can be considered to be in statistical equilibrium with the flow (Arakawa and Schubert 1974; Emanuel et al. 1994), by keeping the Convectively Available Potential Energy (CAPE) con- stant for parcels rising from boundary layer to the midtroposphere. The tropospheric moisture is included as an input to the convection calculation to represent the effect of lateral entrainment of the drier tropospheric air into a rising convective cloud. This acts as a control upon the depth of convection because with a more moist mid-troposphere, updrafts lose less buoyancy from entrainment of environmental air and can reach higher so that for a given amount of lower tropospheric convective heating, the upper tropospheric heating will be greater.

Analyses in Kuang (2008b) identified a moisture-stratiform instability, in addition to the stratiform instability of Mapes (2000). To see it in its most basic form (as illustrated in Fig. 11 of Kuang (2008b)), consider the (horizontal) propagation of a sinusoidal wave with a second-mode temperature structure in the vertical. The propagation of the wave modulates deep (first baroclinic) convective heating by perturbing the statistical equilibrium between the lower troposphere and the subcloud layer. More specifically, deep convection is enhanced behind a warm lower tropospheric temperature anomaly (as large-scale ascent cools the lower troposphere) and is reduced behind a cold lower tropospheric temperature anomaly. The modulations on deep convection peak a quarter cycle behind temperature anomaly peaks. The combined effect of an enhanced deep convective heating and its induced vertical advection of moisture is to moisten the midtroposphere, leading to deeper (i.e. more stratiform) convection in a region where the initial temperature anomalies are positive in the upper troposphere and negative in the lower troposphere, amplifying the initial perturbation. It was also found that in addition to the moist convective damping effect (MCD; e.g. Emanue 1993; Neelin and Yu 1994) where the finite response time of convection damps high frequency waves, the tendency of the second baroclinic mode convective heating to reduce existing 
midtropospheric moisture perturbations tends to reduce the instability at low wavenumbers.

These two effects were suggested as contributors to the wavenumber selection in convectively coupled waves.

This paper describes an extension of the model of Kuang (2008b) to the equatorial beta plane, allowing investigation of the Equatorial Rossby (ER), Mixed Rossby-Gravity (MRG) and Inertio-Gravity (IG) wave types, all of which have significantly different convection/temperature/wind structures and observed amplitudes from the Kelvin-like wave studied in most previous work that is confined to a line along the equator. Our emphasis will be on identifying the basic factors that contribute to the differing instability of the different wave types and wavenumbers.

The linear instability spectrum of the resulting coupled system is found by eigenvalue analysis. We use realistic model parameters estimated from Cloud System Resolving Model (CSRM) studies of the convectively coupled waves. The instability analysis produces unstable waves with phase speeds, growth rates and structures (vertical and horizontal) that compare reasonable well with observations.

The convective parameterization for this model is based upon the quasi-equilibrium concept - that convection responds quickly to the changes in the large-scale flow, so can be considered to be in statistical equilibrium with the flow (Arakawa and Schubert 1974; Emanuel et al. 1994), by keeping the Convectively Available Potential Energy (CAPE) constant for parcels rising from boundary layer to the midtroposphere. The tropospheric moisture is included as an input to the convection calculation to represent the effect of lateral entrainment of the drier tropospheric air into a rising convective cloud. This acts as a control upon the depth of convection, by representing the common observation that, for a given amount of lower tropospheric convective heating, the upper tropospheric heating will be greater if the mid-troposphere is moist.

The linear instability spectrum of the resulting coupled system is found by eigenvalue analysis. We use realistic model parameters estimated from Cloud System Resolving Model (CSRM) studies of the convectively coupled waves. The instability analysis produces unstable waves with phase speeds, growth rates and structures (vertical and horizontal) that compare well with the results from CSRM simulations and observations.

We will show that the instability's dependence upon wave type is related to the projection of mean state Inter-Tropical Convergence Zone (ITCZ) heating onto the mode (as has been suggested in the past: e.g. Takayabu (1994) and Wheeler and Kiladis (1999)) and the an energy feedback efficiency effect. This effect stems from the differing roles of divergent and rotational flows in the convective parameterization and the differing efficiencies that the waves convert wave energy input from convective heating into divergent wind. We investigate this by analytical investigation of a simplified case to explore the details of the mechanisms responsible for wave number and wave type selection in the instability spectrum. The simplified case is chosen to remove the already understood instability mechanisms that exist within the model. Considering the instability in terms of the energy flow between the geopotential and wind fields shows that the instability strength is strongly influenced by the differing efficiencies of conversion from the energy input due to convective heating to divergent winds.

The paper is organized as follows. Section 2 provides a brief description of the model used in this paper. Section 3 describes the results of an eigenvalue analysis conducted to determine the spectrum of unstable waves present in our model, as well as tests of the sensitivity of the 
system to ITCZ configuration and to the parameters used. Section 4 describes a simplified limiting case of our model and an energy flow based feedback mechanism that explains the differing instabilities of the different wave modes. This is followed by a summary and discussion (section 5).

\section{Model description}

a. Model equations

Our basic equations are a simple extension of the model of Kuang (2008b) to the equatorial beta plane. The relation to previous models was discussed in Kuang (2008b) and in the introduction. The model equations can be derived from the anelastic primitive equations for an atmosphere linearized about a resting mean state,

$$
\begin{aligned}
\partial_{t} u^{\prime}-\beta y v^{\prime} & =-\frac{1}{\bar{\rho}} \partial_{x} p^{\prime} \\
\partial_{t} v^{\prime}+\beta y u^{\prime} & =-\frac{1}{\bar{\rho}} \partial_{y} p^{\prime} \\
\partial_{x}\left(\bar{\rho} u^{\prime}\right)+\partial_{y}\left(\bar{\rho} v^{\prime}\right)+\partial_{z}\left(\bar{\rho} w^{\prime}\right) & =0 \\
\partial_{t} T^{\prime}+w^{\prime}\left(\partial_{z} \bar{T}+\frac{g}{c_{p}}\right) & =J^{\prime}-\epsilon T^{\prime} \\
\partial_{z} p^{\prime} & =\frac{g \bar{\rho}}{\bar{T}} T^{\prime}
\end{aligned}
$$

where $\rho$ and $T$ represent the density and temperature, $u, v$, and $w$ represent the velocities in the zonal, meridional and vertical directions respectively and $J$ represents the convective heating. Overbars represent mean quantities and primes denote deviation from this mean state (assumed small). A linear damping on temperature,denoted by $\epsilon$ represents radiative cooling/heating towards the mean temperature profile. The buoyancy frequency, $\mathrm{N}$, is given by:

$$
N^{2}=\frac{g}{\bar{T}}\left(\partial_{z} \bar{T}+\frac{g}{c_{p}}\right)
$$

$\beta y$ is the linear expansion of the Coriolis parameter, $\mathrm{f}$, for small displacements away from the equator.

Then, adding the additional assumptions of a rigid lid and constant buoyancy frequency, we can expand these equations (1-5) in terms of the baroclinic modes numbered $\mathbf{j}$, thusly:

$$
\begin{aligned}
\frac{g \bar{\rho}}{\bar{T}}\left[T^{\prime}, J^{\prime}, w^{\prime}\left(\partial_{z} \bar{T}+\frac{g}{c_{P}}\right)\right] & =\Sigma_{j}[T, J, w]_{j}(x, y, t) \frac{\pi}{2} \sin \left(\frac{j \pi z}{H_{T}}\right) \\
{\left[\bar{\rho} u^{\prime}, \bar{\rho} v^{\prime}, p^{\prime}\right] } & =\Sigma_{j}[u, v, p]_{j}(x, y, t) \frac{H_{T}}{2 j} \cos \left(\frac{j \pi z}{H_{T}}\right)
\end{aligned}
$$

where $H_{T}$ is the height of the lid at the top of the troposphere. We note, as in Kuang (2008b), that despite the above derivation, the use of these modes is based on empirical evidence rather than them being normal modes of the dry atmosphere, as the atmosphere does not possess a rigid lid at the tropopause, a fact emphasized in the context of convectively coupled waves by Lindzen (1974),Lindzen 2003, RaymondFuchs2007)

Substituting the expansion forms (equations (7) and (8)) into our perturbation velocity equations (equations (1)-(3)) yields:

$$
\begin{aligned}
\partial_{t} \overrightarrow{u_{j}} & =-\beta y \hat{k} \times \overrightarrow{u_{j}}+\nabla_{H} T_{j} \\
w_{j} & =-c_{j}^{2} \nabla_{H} \cdot \overrightarrow{u_{j}}
\end{aligned}
$$


where $T$ is the temperature perturbation scaled to act similarly to a geopotential perturbation. $\nabla_{H}$ denotes the horizontal gradient vector operator $\nabla_{H}=\left(\partial_{x}, \partial_{y}\right)$. The wave speeds $c_{j}$ are given by:

$$
c_{j}=\frac{N H_{T}}{j \pi} .
$$

We can also make the modal expansion of the thermodynamic equation (4), together with equation (10), we have:

$$
\partial_{t} T_{j}-c_{j}^{2} \nabla_{H} \cdot \vec{u}_{j}=J_{j}-\epsilon T_{j}
$$

For the rest of this paper, we restrict ourselves to $j=1,2$ - the first two baroclinic modes which are sufficient to explain most of the convective activity (Mapes and Houze 1995). The heating anomalies associated with these two modes, $J_{1}$ and $J_{2}$, represent the convective heating, broken into deep convective and congestus/stratiform components respectively. For the purposes of the convective parameterization, the heating is rewritten in terms of lower (L) and upper (U) troposphere heating anomalies:

$$
\begin{aligned}
L & =\frac{1}{2}\left(J_{1}+J_{2}\right) \\
U & =\frac{1}{2}\left(J_{1}-J_{2}\right)
\end{aligned}
$$

The total upper tropospheric heating is considered to be a fraction of the lower tropospheric heating:

$$
\frac{U_{0}+U}{L_{0}+L}=r_{0}+r_{q} q
$$

where $U_{0}$ and $L_{0}$ are the mean-state heating and $r_{0}$ is the mean state ratio between $L_{0}$ and $U_{0} . \mathrm{q}$ is the mid-tropospheric moisture anomaly and is evolved according to:

$$
\partial_{t} q=a_{1} c_{1}^{2} \nabla_{H} \cdot \overrightarrow{u_{1}}+a_{2} c_{2}^{2} \nabla_{H} \cdot \overrightarrow{u_{2}}-d_{1} J_{1}-d_{2} J_{2}
$$

$a_{j}$ and $d_{j}$ give the mid-tropospheric moisture tendencies due to vertical advection and convection respectively. The effect of horizontal advection of moisture in the midtroposphere is neglected in our formulation. This is not because this advection by the second mode is itself small (first mode advection is approximately zero in our uniformly stratified atmosphere), rather it is neglected in combination with the opposite signed advection above and below. These advection effects are expected to roughly cancel (in their contributions to entrainment drying) allowing us to continue to use a convective parameterization that makes the representation of lower and upper tropospheric moisture difficult to implement.

Regarding the above discussion: In previous studies (ref), the mid-tropospheric moisture anomaly is in general used as a control on precipitation or precipitation efficiency. Inadvertently not discussed in K08, a notable exception is Khoudier and Majda (2006a), which included a nonlinear switch function that favors ?? the effect of free troposphere moisture anomalies. While the physical meaning of their formulation for congestus heating (Eq. 2.8 in Khoudier and Majda (2006a)) is somewhat unclear (congestus heating is relaxed towards the downdraft multiplied by a measure of the dryness of the free troposphere), this formulation does have the effect of modulating the depth of convection based on the free tropospheric moisture anomaly

In a region of the mid-troposphere which is anomalously moist with positive $\mathrm{q}$, the entrained air is more moist than average and the associated cooling is smaller, leading to 
relatively increased buoyancy of parcels in this region. This in turn leads to parcels rising to greater altitude and heating the upper troposphere more. The opposite holds for a region with an anomalously dry mid-troposphere.

The lower tropospheric heating is considered to be relaxing quickly (over a time scale $\tau_{L}$ - typically a few hours) towards the quasi-equilibrium situation given by constancy of lower troposphere non-entraining CAPE. In our context, quasi-equilibrium will be enforced by keeping the difference between the boundary layer moist static energy $\left(h_{b}\right)$ and the vertically averaged lower tropospheric saturation moist static energy $\left(<h^{*}>\right)$ approximately constant. $h_{b}$ and $<h^{*}>$ both vary under the action of convection - a positive convection anomaly dries and cools the boundary layer through the action of downdraughts and modifies the lower tropospheric saturation MSE primarily (in our model) by heating the atmosphere - to reach a consistent equilibrium state. Thus, if equilibrium was to be reached instantaneously:

$$
\partial_{t} h_{b}=\left\langle\partial_{t} h^{*}\right\rangle_{L T}
$$

where LT is the region of the lower troposphere where this QE formulation is assumed to hold - extending vertically up to the level where entrainment begins to become important Assuming that the surface flux anomalies are negligible, we can write

$$
\partial_{t} h_{b}=\left(-b_{1} J_{1}-b_{2} J_{2}\right) .
$$

The $b_{j}$ describe the reduction of boundary layer moist static energy by the convection $J_{j}$.

Meanwhile, we also assume that the averaged saturation moist saturation energy in the lower troposphere can be well approximated by a linear combination of the two baroclinic mode temperature anomalies

$$
\left\langle\partial_{t} h^{*}\right\rangle_{L T}=\partial_{t}\left[F\left(\gamma T_{1}+(1-\gamma) T_{2}\right)\right]
$$

$\mathrm{F}$ is a proportionality constant relating the change in lower-troposphere temperature to the change in moist static energy in the same region. $\gamma$ describes the relative influence of the two modes on temperature of the lower troposphere region. It is, loosely speaking, equivalent to the fractional depth of the troposphere through which this QE formulation can be assumed to hold before entrainment becomes an important effect.

Substituting these two relations into equation (17) allows us to quantify the quasiequilibrium in terms of our model.

$$
\left(-b_{1} J_{1}-b_{2} J_{2}\right)=F\left[\gamma \partial_{t} T_{1}+(1-\gamma) \partial_{t} T_{2}\right]
$$

Expanding this equation using equations (12-15), we can find a lower-tropospheric heating, $L_{e q}$ that satisfies the quasi-equilibrium assumption for the large scale variables $(q, u$, and $T)$.

$$
\begin{aligned}
L_{e q}= & \frac{A L_{0} r_{q}}{B} q-\frac{F \gamma c_{1}^{2}}{B}\left(\nabla_{H} \cdot \overrightarrow{u_{1}}-\frac{\epsilon}{c_{1}^{2}} T_{1}\right) \\
& -\frac{F(1-\gamma) c_{2}^{2}}{B}\left(\nabla_{H} \cdot \overrightarrow{u_{2}}-\frac{\epsilon}{c_{2}^{2}} T_{2}\right),
\end{aligned}
$$

where,

$$
\begin{aligned}
& B=F+\left(b_{1}+b_{2}\right)-A\left(r_{q} q+r_{0}\right) \\
& A=\left(b_{2}-b_{1}\right)+F(1-2 \gamma) .
\end{aligned}
$$


We can improve the realism of the model by assuming that the convection does not respond to changes in the large-scale environment instantly. Rather, we allow $L$ to relax towards $L_{e q}$ over a short timescale $\tau_{L}$ :

$$
\partial_{t} L=\frac{1}{\tau_{L}}\left(L_{e q}-L\right)
$$

By introducing meridional velocity fields $v_{j}$ and the Coriolis force terms, $\beta y \hat{k} \times \overrightarrow{u_{j}}$, this extension prevents the simple reduction of the dry part of the prognostic equations to temperature fields that occurs in Kuang (2008b), leading to the presence of velocity fields in the equations for $L_{e q}$, (21) and $q$, (16) (c.f. equations (23) and (11) of Kuang (2008b)).

\section{b. Parameter Choices}

As described in Kuang (2008b), our parameter values, such as $a_{j}, b_{j}, d_{j}, \mathrm{~F}, \gamma, r_{0}, r_{q}, \epsilon$ and $\tau_{L}$ are estimated from the CSRM simulations of Kuang (2008a) which showed spontaneously developing convectively coupled waves. The estimation is conducted by projecting the CSRM results onto the vertical structure of our modes and interpreting regression results in the framework of our convective parameterization. We consider this approach to be somewhat more rigourous than tuning our model to match observations, and more efficient given the pre-existence of the CSRM results.

Parameter values are given in Table 1 and are essentially identical to those used by Kuang (2008b). The units of the parameters are based upon measuring energies in the equivalent temperature change (in $K$ ) for $1 \mathrm{~kg}$ of dry air gaining that amount of energy. Moisture content is likewise measured by the temperature change that would result if all the stored latent heat in a volume of air was converted to thermal energy in an identical, but dry, air mass

\section{Beta-plane linear instability analysis}

In Wheeler and Kiladis (1999), the observations are of waves of finite amplitude. If the waves are indeed unstable, in the observations the linear growth of the unstable waves is balanced by nonlinear terms and an equilibrium is reached. In this case, the equilibrium is of a statistical nature, with waves growing and dying as they move around and enter regions of differing forcing (due to the changing background state and wave-wave interactions) and is also time varying on various scales. In this paper we investigate the linear regime of infinitesimal waves with a zonally and temporally fixed background state. We see this as a first step toward an understanding of the spectrum. Further investigation of the nonlinear effects is planned. Also planned is an investigation of other interpretations of the observations, such as stable waves continually excited by stochastic or extra-tropical forcing (e.g. Zhang and Webster, 1992; Hoskins and Yang, 2000) in the context of this model.

\section{a. Linearization}

Calculation of the instability spectrum assumes that our system is growing from infinitesimal anomalies, so we can remove any nonlinear terms from the equation set. In our case, linearization is simply achieved by ignoring the first term in the second parentheses from the definition of B (equation 22): 


$$
B=F+\left(b_{1}+b_{2}\right)-A r_{0}
$$

and linearizing equation (15) into:

$$
U=L_{0} r_{q} q+r_{0} L
$$

Our linearized model consists of equations (9), (12), (13), (14), (16), (21), (25), (23), (24), and (26).

For our control experiment, the basic state, represented by the parameter $L_{0}(y)$, is a state of uniform background convection.

The linear system is then Fourier transformed to zonal wave-number/frequency space and the resulting eigenvalue problem is solved on a meridionally discrete domain extending approximately $6000 \mathrm{~km}$ to the north and south, with 181 regularly spaced points. This large domain is utilized to reduce the influence of any edge related errors, and can be implemented without significant computational cost. The resulting complex frequency eigenvalues can be separated into the real part $\omega$, which gives the modal frequency, and the imaginary part $\Gamma$, which gives the modal growth rate. The corresponding eigenvectors give the modal structure in the prognostic fields $\left(u_{1}, v_{1}\right.$ etc). b. Results

c. A realistic mean state $I T C Z$ configuration

To address the question of how the wave structure might be influenced by more realistic ITCZ mean state configuration, we have repeated this experiment several times, using differenty $L_{0}(y)$ to represent different possible ITCZ configurations in the mean state.

Firstly, we use basic state that is an idealization of the zonal mean state. As shown in Figure 2, this lower tropospheric heating profile consists of a Gaussian peak in mean state convection centered on the equator, with a width of $5^{\circ}$, above a small, nonzero uniform offset that extends to the edges of the domain.

The results from the linear analysis are shown in figures 3 and 4. Unstable waves are indicated in the frequency/wavenumber diagram, with the marker radius proportional to the growth rate. Unstable modes are assigned, subjectively, to an equivalent dry class (dispersion curve ranges shown dashed) based upon their position in Figure 3, relative to the dry dispersion curves indicated. Investigation of the modal structure (discussed below) shows that these identifications are warranted. The system contains unstable modes corresponding to the Kelvin waves, the $\mathrm{n}=1$ and $\mathrm{n}=2$ Inertio-Gravity waves and the Mixed Rossby-Gravity wave.

Kelvin waves are the most unstable wave type. Wavelengths between about $13000 \mathrm{~km}$ and $2300 \mathrm{~km}$ (planetary wave-numbers 3 and 17) are unstable, with a maximum growth rate of $\sim 0.08 d_{a y}{ }^{-1}$ occurring at wavelength of around $5700 \mathrm{~km}$ (wavenumber 7 ). These waves have phase speeds between $20 \mathrm{~m} / \mathrm{s}$ and $25 \mathrm{~m} / \mathrm{s}$, slightly slower than the second baroclinic wave, with the speed deficit becoming stronger as the wavenumber decreases. The growth 
rate curve rises sharply between wave numbers 3 and 7 , then decays more slowly as the wave-number is further increased to 17 .

Figure 5 shows the structure of this mode, along with vertical structure of temperature. The first baroclinic mode is approximately in quadrature with the second, leading to a tilted vertical temperature structure, similar to observations. Zonal winds blow in the standard dry wave directions. The meridional wind has a small non-zero component. This is not unexpected, as the moist physics that couples our system mixes the modes together so that the convectively coupled "Kelvin" wave observed, while dominated by a Kelvin wave profile, will still contain a small amount of other waves in superposition with it. The wind fields are plotted for the lower troposphere at $z=0$.

Figure 3 shows unstable MRG, also refered to as $\mathrm{n}=0$ EIG waves for the $k>0$ part of the spectrum, (approximately between wavenumber -15 and 10, with peak growthrate of $\sim 0.03$ /day occurring at wavenumber $3-\sim 13000 \mathrm{~km}$ wavelength). The phase speed of the MRG also appears drop below the dry speed as the wavenumber decreases, although this become less visible at large negative wave numbers as the dispersion curves converge. The modal structure of the (eastward) planetary wavenumber 7 Mixed Rossby-Gravity wave is shown in figure 6 .

The system also shows instability in the $\mathrm{n}=1$ (East and West bound) IG waves.

The $\mathrm{n}=2$ IG modes are only marginally unstable in this model. We consider growth rates below a few percent of the peak values to be only marginally unstable and far too dependent on assumptions about parameters to draw solid conclusions about. The sensitivity of our model to parameter variation is explored in section e below.

The reconstruction of the planetary wavenumber 7 Eastward Inertio-Gravity wave is shown in Figure 7. In figure 8, the $k=-7$ Westward Inertio-Gravity wave structure is also shown for comparison. These modes too show horizontal and vertical structures that compare well with observations.

All the reconstructed modes show first and second mode winds with similar amplitudes, broadly consistent with observations (see, for example, Haertel and Kiladis (2004)), as well as a vertically tilted structure, again generally consistent with observations (see, for example, Straub and Kiladis (2001))

Most noticeably absent from the instability spectrum are the Equatorial Rossby Waves and the Madden-Julian Oscillation (MJO). The complete absence of the MJO signal indicates, not unexpectedly, that the current simple model is missing the physics responsible for the MJO. The Rossby waves exist in our system but are stable within a range of parameter choices around our nominal values (discussed below in section e). As both these missing wave types are at similar, low frequencies, the model may have either a too strong damping at low frequencies, or may be missing some other physics that destabilizes waves at these frequencies.

Comparison with Figure 1 shows:

1. Peak Kelvin wave growth occurs at a wavelength comparable to the most active waves in the observations.

2. The ranges of wave number and wave type showing instability in our model match the ranges of waves that are observed in the OLR signals.

3. The Kelvin Wave dispersion curves shifts to higher equivalent depth at higher wavenumbers, opposite to the trend observed. 
4. The model possesses a preference for unstable westward n=1,2 IG modes (as opposed to eastward $\mathrm{n}=1,2 \mathrm{IG})$, similar to that observed.

5. The waves posses a tilted vertical structure that is generally consistent with the observations, with low level temperature anomalies preceeding a brief period of deep anomaly followed by a period of "stratiform" temperature anomaly.

\section{d. Varying ITCZ configurations}

To address the question of whether the dominance of the Kelvin wave is due to the colocation of the mean state heating and the center of the Kelvin wave profile, we have conducted several additional experiments with different ITCZ configurations in our mean state.

Figure 9 shows a double ITCZ mean state with the two peaks approximately a second baroclinic deformation radius north and south of the equator, colocated approximately with the MRG temperature anomaly peaks determined from Figure 6. Unstable modes for this case are shown in Figure 10. While moving the peak heating away from the equator does reduce the overall growth rate of the unstable Kelvin waves and increases that of the unstable MRG waves, the growth rates of the Kelvin waves are still strongest. The MRG growth rates surpass the $\mathrm{n}=1 \mathrm{IG}$ wave in the small wavenumber region of the spectrum. The reconstructed mode structures (not shown) are not significantly different from the single ITCZ case.

Two further experiments (not shown) placed i) a double ITCZ at the off-equatorial peaks of the $\mathrm{n}=1$ IG temperature anomaly field and ii) a single ITCZ at the northern MRG peak temperature anomaly position. While the wave that had the greatest overlap with the convective envelope showed some enhancement of growth rate, neither of these experiments altered the general dominance of the Kelvin waves.

Another aspect of the observed activity profile that stands out as possibly being related to ITCZ configuration is the asymmetry between Eastward and Westward Inertia-Gravity waves - the observed activity of the WIG is much stronger than that of the EIG. It has been suggested that this is primarily due to the fact that the latitudinal structure of the EIG is significantly more complex and a greater proportion of the EIG temperature anomaly is located away from the equator and the ITCZ (see, for example,Takayabu (1994) and Wheeler and Kiladis (1999)). In order to explore this possibility, a further experiment is shown, in which the ITCZ was widened by a factor of 10 and reduced in amplitude by the same factor (to keep the total heating by $L_{0}$ constant). In this situation, the projection of the ITCZ heating profile onto the EIG and WIG structures should be essentially the same. The results are shown in figure 11, with the $\mathrm{n}=1$ IG modes indicated. As can be seen some, but not all, of the east-west asymmetry has been removed. Further experiments (not shown) with even broader ITCZs (essentially flat across the model's meridional extent) showed no significant increases in symmetry. It will be demonstrated below that some of this asymmetry is due to the differing efficiencies with which the East- and West-ward IG waves convert convective heating into more convective heating.

\section{e. Parameter Sensitivity}

As mentioned above, the model does display some sensitivity to parameter variations. Figure 12 shows the growth rate curves for sensitivity experiments conducted with the damp- 
ing $(\epsilon)$ and the convective equilibrium relaxation time $\left(\tau_{L}\right)$ increased and decreased by $10 \%$ The instability for the Kelvin, Equatorial Rossby, Mixed Rossby-Gravity and n=1 InertioGravity are seen to be generally robust, with the location of the peak instabilities in these cases remaining approximately the same. The peak growth rates do change slightly, as is to be expected, when the damping is varied. However, the $\mathrm{n}=2$ and higher IG modes can be observed to vary greatly in their instability character in this range of parameters. As would be expected from the moist convective damping theory, decreasing the convective relaxation time decreases the moist convective damping effect, which then decreases the damping applied to high wavenumber instabilities, leading to an increase in instability for the $\mathrm{n}=2$ and n=3 IG modes. For the same reason, the Kelvin wave peak instability can be seen to shift very slightly to higher frequencies when $\tau$ is decreased.

A similar sensitivity test was applied to all of the semi-empirical paramters contained within our model. Generally, for a $10 \%$ change in parameter value, variations in the spectrum were similar in character and amount to those discussed for damping above (not shown). A few parameters showed interesting sensitivity:

- $r_{0}$ : when $r_{0}$ is varied, the position of the peak instability shifts to higher wavenumber for higher $r_{0}$. The reverse holds for reduced $r_{0}$.

- $c_{2}$ : not surprisingly, as the unstable modes are dominated by the second baroclinic component, a variation in the $c_{2}$ parameter leads to the obvious variation in the speed of the unstable modes. Any unstable mode speed variation caused by modification of $c_{1}$ is negligible

The Equatorial Rossby waves are still not observed under any of these parameter varia- tions

These results are similar to the more detailed sensitivity study conducted for the 1D version of this model included in Kuang (2008b).

\section{The role of energy flow efficiency}

This model has previously been analyzed for Kelvin-like waves on the equator (Kuang 2008b), revealing some of the mechanisms determining the instability spectrum. Specifically, the MCD effect (e.g. Emanuel 1993; Neelin and Yu 1994) damps high frequency waves, and the tendency of the second baroclinic mode convective heating to reduce existing midtropospheric moisture perturbations tends to reduce the instability at low wavenumbers. Expansion of the model to the beta plane in this paper has revealed the existence of at least one more mechanism within the model, responsible for the differences in growth rates for waves with similar frequencies. We have already shown that the ITCZ configuration and projection of the mean state heating on to the modes' temperature anomaly profiles exert some control upon the shape of the growth rate curves. However, this control is less than might be expected and does not seem to explain all the aspects. We hypothesize that the growth rate curves for the various wave types are further shaped by the relative efficiency with which the various waves convert input energy into the divergent winds, which in turn generate more energy input. In this section we will show that the growth rates can be directly linked to the generation of divergent winds in the modes. In order to explore the mechanisms that control the shape of the instability spectrum, in this section of the paper we consider a simplified system that has been modified to remove the mechanisms already identified in 
Kuang (2008b) and any ITCZ convective projection amplitude effects (discussed in section

$3 \mathrm{c}$, above), through the choice of specific parameters. Some more detailed exploration of limiting cases within the model (in 1D) is conducted in Kuang (2008b).

\section{a. Physical Description of simplified system}

Our simplified system is based upon the second limiting case of Kuang (2008b), where the convective mass flux is dominated by entraining parcels and the boundary layer is in quasi-equilibrium with the second mode temperature $(\gamma=0)$. The first temperature mode is essentially uncoupled from our system and can be ignored. The system is further simplified by setting $b_{2}=0$ to remove the influence of the second mode heating upon the boundarylayer moist static energy and $\epsilon=0$ to remove the radiative damping. $d_{2}$ is also set to zero to remove the effect of second mode heating upon the mid-tropospheric moisture, $q$. These changes remove the scale selection of the moisture-stratiform instability

Let us further tighten quasi-equilibrium to "strict quasi-equilibrium" by enforcing instantaneous relaxation to the equilibrium values of $\mathrm{L}$ (by setting $\tau_{L}=0$ ). This change remove the MCD effect.

A uniform $L_{0}$ profile is used, removing the ITCZ projection effects.

Importantly, the parameter $r_{q}$, which controls the coupling between moisture and heating is set to a very small value, so that the heating can be approximated as an infinitesimal perturbation upon the dry system.

This modified case is evaluated numerically (Figure 13) in the same manner as the control case. The simplified system shows many unstable modes, but for this section, we will concentrate on the Kelvin, MRG, $\mathrm{n}=1 \mathrm{ER}$ and $\mathrm{n}=1 \mathrm{IG}$ modes, so the modes with $n>1$ are deliberately omitted in Figure 13. The Kelvin wave has a constant growth rate for al wave numbers greater than zero. The MRG wave growth rate increases monotonically from the very small for large negative wave numbers, to relatively large at high positive wave numbers, with rapid increase in the region between $k=-5$ and $k=+5$. In the domain evaluated, the MRG growth rate never reaches that of the Kelvin wave. The system also shows unstable ER and IG waves, with ER growth rates increasing from a low value for large (negative) wave numbers to close to the Kelvin value for wavenumber zero. $n=1$ IG waves have a large growth rate for all wave numbers, with growth increasing as $|k|$ increases. (Discuss $\mathrm{k}=0$, omega $=0$ mode)

\section{b. Energy flow from heating into divergent winds and heating feedback}

In order to demonstrate our hypothesis, let us consider how the energy that flows into the wave due to our convective heating goes into increasing the divergence of the flow. As we will show, the amplitude of the convective heating is proportional to the amplitude of the divergence, so this component of the energy drives the positive feedback, whereas energy that flows into the rotational component or the potential energy of the wave is essentially trapped there and cannot contribute to the further growth of the wave.

For this section, let us consider a wave that is essentially dry in character, that is forced by a diagnostic heating that approximates the simplified case discussed above. We will further consider how this wave changes with time over a short interval while the heating acts. 
The heating can be derived as an approximately diagnostic function of the dynamical variables as follows:

In our limiting case, equations (16) and (20) become:

$$
\begin{aligned}
\partial_{t} q & =\left(a_{1}-d_{1}\right) J_{1} \\
-b_{1} J_{1} & =F \partial_{t} T_{2}
\end{aligned}
$$

where we have further assumed that the vertical velocity field is well approximated by the heating field (a good assumption for the first mode heating, as confirmed by the modal eigenstructures for the control model discussed above). Equation $27(* * *)$ states the combined effect of deep convection and the associated vertical moisture advection is to moisten the mid-troposphere. Equation $28(* * *)$ is a statement of deep convection maintaining strict quasi-equilibrium between the boundary layer and the second baroclinic temperature mode.

Furthermore, we can also write (from equations (13), (14) and (15)):

$$
J_{2}=-r_{q} L_{0} q
$$

(letting $r_{0}=1$ ).

Combining the above equations we have:

$$
\begin{aligned}
\partial_{t} J_{2} & =-r_{q} L_{0} \partial_{t} q \\
& =-r_{q} L_{0}\left(a_{1}-d_{1}\right) J_{1} \\
& =\frac{r_{q} L_{0}\left(a_{1}-d_{1}\right) F}{b_{1}} \partial_{t} T_{2}
\end{aligned}
$$

We can see that $J_{2}$ keeps the same phase as $T_{2}$. Further, as $J_{2}$ is a very small perturbation upon the dry wave, we can also write:

$$
\partial_{t} T_{2} \approx c_{2}^{2} \delta_{2}
$$

where the divergence, $\delta_{2}$ is defined by:

$$
\delta_{2}=\nabla_{H} \cdot \overrightarrow{u_{2}}
$$

and thus

$$
\partial_{t} J_{2}=\frac{r_{q}\left(a_{1}-d_{1}\right) F c_{2}^{2}}{b_{1}} \delta_{2}
$$

So we have:

$$
\left|J_{2}\right| \propto \frac{\left|\delta_{2}\right|}{\omega}
$$

Combining our above relations for the heating field, we define:

$$
J_{2}=-\frac{B\left|\delta_{2}\right| T_{2}}{\omega\left|T_{2}\right|}
$$

$\mathrm{B}$ is a constant of proportionality, considered to be very small, so that the heating is only a small perturbation to the waves.

$$
B=-\frac{r_{q}\left(a_{1}-d_{1}\right) F c_{2}^{2}}{b_{1}}
$$

In this limiting case, our equation set reduces to: 


$$
\begin{aligned}
& \partial_{t} u_{2}=\beta y v_{2}+\partial_{x} T_{2} \\
& \partial_{t} v_{2}=-\beta y u_{2}+\partial_{y} T_{2} \\
& \partial_{t} T_{2}=c_{2}^{2} \delta_{2}+J_{2} .
\end{aligned}
$$

From this point, the first mode is completely superfluous, so we can drop the subscripts without ambiguity.

1) Kelvin Wave heating-divergence feedback

We start with a neutral Kelvin Wave, with the standard form (assuming the dry dispersion relationship to be sufficiently accurate given the arbitrarily small heating) for the complex wave:

$$
\begin{aligned}
\tilde{u} & =u_{0} e^{\left(-\frac{\beta y^{2}}{2 c}\right)} e^{i(k x-\omega t)} \\
\tilde{v} & =0 \\
\tilde{T} & =-c^{2} \frac{k}{\omega} u_{0} e^{\left(-\frac{\beta y^{2}}{2 c}\right)} e^{i(k x-\omega t)} \\
& =c u_{0} e^{\left(-\frac{\beta y^{2}}{2 c}\right)} e^{i(k x-\omega t)}
\end{aligned}
$$

where the amplitude of $\tilde{T}$ is determined using the standard wave relations (in wavenumber/frequency space) and the dry dispersion equation (see, for example, Gill (1982)).

We can then calculate the total energy (per unit length in the $x$-direction) stored in the wave ( $\mathrm{H}$ is the equivalent depth of the second mode):

$$
\begin{aligned}
E & =\frac{1}{2} \overline{u^{2}} H+\frac{1}{2} \frac{\overline{T^{2}}}{g} \\
& =\frac{H}{2}\left(u_{0}^{2}+\frac{\left(c u_{0}\right)^{2}}{c^{2}}\right)\left(\overline{e^{\left(-\frac{\beta y^{2}}{c}\right) \cos ^{2}(k x-\omega t)}}\right) \\
& =H u_{0}^{2} \frac{\omega}{2 \pi} \frac{k}{2 \pi} \int_{0}^{\frac{2 \pi}{\omega}} \int_{0}^{\frac{2 \pi}{k}} \cos ^{2}(k x-\omega t) d x d t \int_{-\infty}^{\infty} e^{-\left(\frac{\beta y^{2}}{c}\right)} d y \\
& =\frac{H}{2} \sqrt{\frac{c \pi}{\beta}} u_{0}^{2}
\end{aligned}
$$

The overbar denotes the time and zonal mean of a quantity, integrated over its meridional extent.

The energy input in time $\Delta t$ is given by:

$$
\begin{aligned}
\Delta E & =\overline{J T} \Delta t \\
& =\frac{\overline{-B|\delta| T^{2}}}{\omega|T|} \Delta t \\
& =\frac{B|\delta|}{\omega c u_{0}} c^{2} u_{0}^{2} e^{-\left(\frac{\beta y^{2}}{c}\right)} \cos (k x-\omega t) \Delta t \\
& =\frac{B|\delta| c u_{0} \Delta t}{2 k} \sqrt{\frac{c \pi}{\beta}}
\end{aligned}
$$

$\left(|T|=-c u_{0},|\delta|\right.$ is defined similarly)

With the increased energy in the wave after this interval, the amplitude of the wave (given by $u_{0}$ ) will have increased:

$$
\begin{aligned}
\Delta u_{0} & =\left(\partial_{u_{0}} E\right)^{-1} \Delta E \\
& =\frac{B c|\delta| \Delta t}{2 H k}
\end{aligned}
$$

Now, the divergence in the wave can also be determined: 


$$
\delta=\partial_{x} u=-k u_{0} e^{-\left(\frac{\beta y^{2}}{2 c}\right)} \sin (k x-\omega t) .
$$

We can calculate how the divergence increases in this time interval:

$$
\Delta \delta=-k \Delta u_{0} e^{-\left(\frac{\beta y^{2}}{2 c}\right)} \sin (k x-\omega t) .
$$

Because $\Delta \delta$ is in exact phase with $\delta$ we can also say that:

$$
\begin{aligned}
\Delta|\delta| & =k \Delta u_{0} \\
& =k \frac{B c|\delta| \Delta t}{2 H k} \\
\frac{\Delta|\delta|}{\Delta t} & =\frac{B c}{2 H}|\delta|
\end{aligned}
$$

As shown, the amplitude of the Kelvin Wave divergence grows exponentially with time, at a rate that is independent of wavenumber, as observed in the numerical analysis above.

2) Mixed Rossby-Gravity wave heating-Divergence feedback

The basic dry MRG wave is well described by its y-velocity field:

$$
\tilde{v}=v_{0} e^{-\left(\frac{\beta y^{2}}{2 c}\right)} e^{i(k x-\omega t)} .
$$

and a dispersion relationship:

$$
\omega= \pm \frac{k c}{2}\left(1 \pm \sqrt{1+\frac{4 \beta}{k^{2} c}}\right)
$$

Then, using the standard forms:

$$
\begin{aligned}
\tilde{T} & =-\frac{i \omega c^{2}}{\omega^{2}-c^{2} k^{2}}\left(-\partial_{y}+\frac{k}{\omega} \beta y\right) \tilde{v} \\
& =\frac{i c v_{0} \beta y e^{-\left(\frac{\beta y^{2}}{2 c}\right)}}{c k-\omega} e^{i(k x-\omega t)} \\
\tilde{u} & =\frac{\beta y \tilde{v}+i k \tilde{T}}{-i \omega} \\
& =-\frac{i v_{o} \beta y e^{-\left(\frac{\beta y^{2}}{2 c}\right)}}{c k-\omega} e^{i(k x-\omega t)}
\end{aligned}
$$

Similarly to the Kelvin Wave example, we can find the energy per unit length stored in this wave:

$$
E=\frac{H}{4} \frac{v_{0}^{2}\left(c\left(c k^{2}+\beta\right)-2 c k \omega+\omega^{2}\right)}{(\omega-c k)^{2}} \sqrt{\frac{\pi c}{\beta}} .
$$

Then, the increase in the wave amplitude due to an increase in energy $\Delta E$ is given by:

$$
\Delta v=\frac{2 \Delta E(\omega-c k)^{2}}{H v_{0}\left(c\left(c k^{2}+\beta\right)-2 c k \omega+\omega^{2}\right)} \sqrt{\frac{\beta}{\pi c}},
$$

and the increase in energy over $\Delta t$ is:

$$
\Delta E=\overline{J T}=\frac{B c^{4} v_{0}|\delta| \Delta t}{4 \beta \omega(\omega-c k)} \sqrt{\frac{\pi c}{\beta}},
$$

$\left(|T|=c v_{0} \beta /(c k-\omega),|\delta|\right.$ is defined similarly $)$.

leading to an increase in the wave amplitude of 


$$
\Delta v=\frac{B c^{3}|\delta| \Delta t(\omega-c k)}{2 H \omega\left(c\left(c k^{2}+\beta\right)-2 c k+\omega^{2}\right)} .
$$

The divergence of the MRG wave is

$$
\delta=\frac{v_{0} \omega \beta}{c^{2} k-c \omega} y e^{-\left(\frac{\beta y^{2}}{2 c}\right)} \cos (k x-\omega t)
$$

and the magnitude of the divergence is

$$
|\delta|=\frac{v_{0} \beta \omega}{c(\omega-c k)}
$$

(note the change of sign so that the magnitude of the divergence is positive - this simplifies interpretation).

$$
\begin{aligned}
\frac{\Delta|\delta|}{\Delta t} & =\frac{B c}{2 H}|\delta|\left(\frac{c \beta}{c\left(c k^{2}+\beta\right)-2 c k \omega+\omega^{2}}\right) \\
& =\frac{B c}{2 H}|\delta| \frac{\beta}{2 \beta \mp \frac{k^{2} c}{2}\left(\mp 1+\sqrt{1+\frac{4 \beta}{k^{2} c}}\right)}
\end{aligned}
$$

where the upper sign applies for positive wave numbers and the lower sign applies for negative wave numbers.

3) Equatorial Rossby and InERTio-Gravity WAVE HEATING-DivergenCE FEedBACK

A similar analysis for the $\mathrm{n}=1$ Rossby and Inertio-Gravity waves yields:

$$
\frac{\Delta|\delta|}{\Delta t}=\frac{B c}{2 H}|\delta|\left(\frac{c\left(3 c^{2} k^{2}+2 c k \omega_{n=1}+2 \omega_{n=1}^{2}\right)}{c^{4} k^{4}+3 c^{3} k^{2} \beta+3 c \beta \omega_{n=1}^{2}+\omega_{n=1}^{4}+2 c^{2} k \omega_{n=1}\left(\beta-k \omega_{n=1}\right)}\right)
$$

where

$$
\omega_{n=1} \approx\left\{-\frac{\beta k}{k^{2}+3 \frac{\beta}{c}}, \sqrt{k^{2} c^{2}+3 \beta c}\right\}
$$

for the Rossby and IG waves respectively (see, for example, Gill (1982)) obtained from the dispersion relationship

$$
\left(\frac{\omega}{c}\right)^{2}-k^{2}-\beta \frac{k}{\omega}=3 \frac{\beta}{c}
$$

by ignoring the first and last terms on the left hand side respectively.

\section{c. Energy feedback results}

Figure (14) shows the growth rate of the MRG, $n=1$ ER and $n=1$ IG waves in this construction, normalized by the constant growth rate of the Kelvin waves. The growth rate of the MRG increases as the divergent component of the wave increases from a small value at negative wave numbers (where the wave is very much like the low-divergence ER waves) to a large value at positive wave numbers, where the wave most resembles the high-divergence Kelvin wave. Just as observed in the numerical study (Figure 13), the growth rate increases rapidly between $k=-5$ and $k=+5$

The growth rate of the $n=1$ ER wave increased as $k$ increases from $-\infty$ to zero, as observed in the numerical results. The growth rate of the $\mathrm{n}=1 \mathrm{IG}$ wave increases as $|k|$ increases, from a small non-zero value at $k=0$ as observed.

It is interesting to note that the Rossby waves achieve growth rates very close to those of the Kelvin waves, as the Rossby waves are typically considered to be dominated by 
vorticity. However, this can be explained by visualizing a very long wavelength Rossby wave $(k \approx 0$ - refer left column of Figure 15). This wave will consist of nearly uniform motion away from and towards the equator, with zonally uniform meridional velocity and near zero zonal velocity. In this case, the wave clearly has a large divergence and very small vorticity. As the wavenumber increases, the contribution of the zonal divergence increases, offsetting the meridional divergence (due to the phase difference). At large wave numbers, the two components cancel, leading to reduced divergence and a wave has significantly larger rotational flow (right column of Figure 9).

The Inertio-Gravity wave spectrum is explained by similar logic, although the details are different due to the different structures of the waves, particularly the non-zero vorticity at zero wavelength and the increasing divergence with wavelength.

The numerically calculated values of the corresponding growth rates (also normalized to the numerical Kelvin wave growth rate) are also plotted on this Figure for comparison. The MRG and ER growth rates are essentially indistinguishable from the analytic solutions. The discrepancy for the inertio-gravity waves stems from the approximation used for the wave frequency in equation (62), which is only accurate to within $13 \%$ in this case (see, for example, Gill (1982)), whereas the error in the ER frequency is less than $2 \%$.

The physical content of this argument is contained more in the method than the final expressions. The construction of instability mechanism here emphasizes the differing efficiencies with which the modes can convert input energy into divergent flow, driving more energy input. As the growth rates derived from this construction of the model resemble the numerical results closely, we feel that this vindicates our hypothesis, illuminating a further mechanism that influences the linear instability spectrum of the equatorial convectively coupled waves.

The specifics of our derivation assume that the basic instability mechanism relies upon low level divergence to drive convective heating. The detailed result is thus dependent upon the existence of the moisture-stratiform (or similar) instability mechanism.

\section{Summary and Discussion}

In this paper, we have demonstrated that our extension of the model of Kuang (2008b) to the equatorial beta plane allows us to produce a linear system with a spectrum of unstable modes that bear a good resemblance to the modes visible in the global OLR data, in terms of excited wavenumber range and also in terms of the wave types present. The unstable Kelvin, MRG, and IG waves visible in the results of Wheeler and Kiladis (1999) are reproduced well using this model.

As discussed in the introduction, simple models and interpretations of the convectively coupled waves date back as least as far as Lindzen (1974). In this paper, Lindzen considers the heating to be driven purely by convergent flow lifting conditionally unstable parcels high enough that they begin to convect, which leads to warming of the troposphere and reenforces the convergent flow (known as wave-CISK). This mechanism shares some characteristics with the energy feedback arguments we made above, in that the growth of modes is related to how strong the divergent winds of that mode are - for example, Lindzen determines that the East-West asymmetry in the $n=1,2$ IG waves may be related to the differing amounts of convergence in the different propagation directions.

Our moisture-stratiform instability mechanism, however, is very different from wave- 
CISK; by assuming quasi-equilibrium, enhanced deep convection in our model leads to cooling of the troposhere by cooling and drying the boundary layer. A purely wave-CISK mechanism suffers from an "Ultra-Violet Catastrophe" in which the energy at ever shorter wavelengths increases and the total energy in the spectrum diverges. In contrast, the moisturestratiform instability has bounded growth rates at short wavelengths and the MCD effect further cuts off the wave energy at short wavelengths, averting the catastrophe.

Other interprations of the spectrum, like those of Takayabu (1994) and Wheeler and Kiladis (1999) use the differing projections of the mean state heating onto the wave structure to explain the East-West asymmetry. In the context of our model, we have shown that the asymmetry still exists even with a meridionally uniform mean state heating.

Our model is closely related to that of Khouider and Majda (see, for example, Khoudier and Majda (2006a), Khoudier and Majda (2006b), and Khouider and Majda (2008)), especially in the inclusion of mid-tropospheric moisture as a control of convection depth. However, by making our model conceptually simpler than their model, the various instability and spectrum shaping mechanisms are made clearer. The further extension of our model to the beta plane also allows the investigation of instability shaping due to the mean state profile and due to the meridional winds of the various waves observed on the equatorial beta plane.

The model does fail to produce any unstable ER or MJO waves. As stated above, this may be due to our model possessing too great a low frequency damping or lacking an effect that serves to destabilize the low frequency waves. It is also possible that the lack of Moisture (for example Raymond and Fuchs (2007)) or WISHE-type surface flux anomaly driven (either due to surface wind or solar irradiation anomalies, see for example?) modes within our model may cause the absense of these modes. However, the observations of Yang et al. (2007) indicate that the Rossby wave signal detected in the OLR spectrum might have a different instability character. For example, they may be unstable only to finite disturbances, requiring a large extra-tropical Rossby or near-resonant MRG wave to excite them. Or possibly, the waves are simply stable with their decay in balance to near constant stochastic forcing from the extratropics and the MRG activity. The lack of MJO activity is possibly also related to the lack of non-linear effects in our model. The non-linear time evolution of this model is a subject that we intend to address in the near future.

It was observed that the position and size of the ITCZ affects the growth rates of the different waves: modes that posses a large spatial overlap with the ITCZ profile are enhanced while those with a small overlap are weaker. However, even when the ITCZ configuration provides large overlap for the MRG or IG waves and small overlaps for the Kelvin waves, the Kelvin wave growth rates remain large compared to those of the MRG and IG waves indicating additional factors affect the growth rates of different wave types. We suggest that the efficiency with which waves convert input energy into divergent winds is an important factor.

With the addition of our energy-flow divergence feedback mechanism, the basic shape of the wave spectrum is starting to become clearer. The linear part of the spectrum is shaped by the combined actions of moist convective damping, which damps high frequency waves, and the damping effect of the second mode convective heating on midtropospheric moisture anomalies damps more strongly the lowest frequency waves, the ITCZ projection effect, and the energy-flow divergence feedback which selects the wave types with larger divergent components. 
Analytical extension of the energy flow feedback to our full set of equations is expected to be non-trivial; furthermore, our discussion is based on a simple linear model of convectively coupled waves. However, it seems likely that the principle of "wasted energy" in the rotational component of the velocity fields of the equatorial waves will also apply in the real atmosphere, helping to shape the observed spectrum of convectively coupled equatorial waves.

\section{Acknowledgments}

This work was partly supported by the Modeling, Analysis and Prediction (MAP) Program in the NASA Earth Science Division. The authors would like to thank William Boos, David Romps and Christopher Walker for helpful comments and suggestions during the drafting of this paper. Comments by Matthew Wheeler, David Raymond and an anonymous third reviewer also improved the paper

\section{REFERENCES}

Arakawa, A. and W. H. Schubert, 1974: Interaction of a cumulus cloud ensemble with the large-scale environment, part i. J. Atmos. Sci., 31, 674-701.

Arkin, P. A. and P. E. Andanuy, 1989: Estimating climatic-scale precipitation from space: A review, J. Climate, 2, 1229-1238.

Chang, C. P., 1970: Westward propagating cloud patterns in the tropical pacific as seen from time-composite satellite photographs. J. Atmos. Sci., 27, 133-138.

Emanuel, K. A., J. D. Neelin, and C. S. Bretherton, 1994: On large-scale circulations in convecting atmospheres. Quart. J. Roy. Meteor. Soc., 120, 1111-1143.

Gill, A. E., 1982: Atmosphere-Ocean Dynamics. Academic Press, 438-439 pp.

Haertel, P. T. and G. N. Kiladis, 2004: Dynamics of 2-day equatorial waves. J. Atmos. Sci, $61,2707-2721$.

Khoudier, B. and A. J. Majda, 2006a: Multicloud convective parameterizations with crude vertical structure. Theor. Comput. Fluid Dyn., 20, 351-375.

Khoudier, B. and A. J. Majda, 2006b: A simple multicloud parameterization for convectively coupled waves. part i: Linear analysis. J. Atmos. Sci, 63, 1308-1323.

Khouider, B. and A. J. Majda, 2008: Multicloud models for organized tropical convection Enhanced congestus heating. JAS, 65, 895-914. 
Kuang, Z., 2008a: Modeling the interaction between cumulus convection and linear waves in a limited domain cloud system resolving model. J. Atmos. Sci., 65, 579-591.

Kuang, Z., 2008b: A moisture-stratiform instability for convectively coupled waves. J. Atmos. Sci., 65, 834-854.

Liebman, B. and C. A. Smith, 1996: Description of a complete (interpolated) outgoing longwave radiation dataset. $B A M S, 77,1275-1277$.

Lindzen, R. S., 1974: Wave-cisk in the tropics. J. Atmos. Sci., 31, 156-179.

Majda, A. J. and M. G. Shefter, 2001: Models for the stratiform instability and convectively coupled waves. J. Atmos. Sci., 58, 1565-1584.

Mapes, B. E., 2000: Convective inhibition, subgrid-scale triggering energy, and stratiform instability in a toy tropical wave model. J. Atmos. Sci., 57, 1515-1535.

Mapes, B. E. and R. A. Houze, 1995: Diabatic divergence profiles in western pacific mesoscale convective systems. JAS, 52, 1807-1828.

Matsuno, T., 1966: Quasi-geostrophic motions in the equatroial area. J. Meteor. Soc. Japan, $44,25-43$.

Nakazawa, T., 1988: Tropical super clusters within intraseasonal variations over the western pacific. J. Meteor. Soc. Japan, 66, 777-786.

Neelin, J. D., I. M. Held, and K. H. Cook, 1987: Evaporation-wind feedback and low frequency variability in the tropical atmosphere. J. Atmos. Sci., 44, 2341-2348.
Raymond, D. J. and Z. Fuchs, 2007: Convectively coupled gravity and moisture modes in a simple atmospheric model. Tellus A, 59, 627-640.

Straub, K. H. and G. N. Kiladis, 2001: Observations of a convectively coupled kelvin wave in the eastern pacific itcz. J. Atmos. Sci., 59, 30-53.

Straub, K. H. and G. N. Kiladis, 2002: Observations of a convectively coupled kelvin wave in the eastern pacific itcz. J. Atmos. Sci., 59, 30-53.

Takayabu, Y. N., 1994: Large-scale cloud disturbances associated with equatorial waves. part ii: Westward-propagating inertio-gravity waves. JMSJ, 72, 451-465.

Tulich, S. N. and B. E. Mapes, 2008: Multiscale convective wave disturbances in the tropics: Insights from a two-dimensional cloud-resolving model. J. Atmos. Sci., 65, 140-155.

Tulich, S. N., D. A. Randall, and B. E. Mapes, 2007: Vertical-mode and cloud decomposition of large-scale convectively coupled gravity waves in a two-dimensional cloud resolving model. J. Atmos. Sci., 64, 1210-1229.

Wang, B., 1988: Dynamics of tropical low-frequency waves - an analysis of the moist kelvin wave. J. Atmos. Sci., 45, 2051-2065.

Wheeler, M. and G. N. Kiladis, 1999: Convectively coupled equatorial waves: Analysis of clouds and temperature in the wavenumber-frequency domain. J. Atmos. Sci., 56, 374 399

Wheeler, M., G. N. Kiladis, and P. J. Webster, 2000: Large-scale dynamical fields associated with convectively coupled equatorial waves. J. Atmos. Sci., 57, 613-640. 
Yang, G. Y., B. Hoskins, and J. Slingo, 2007: Convectively coupled equatorial waves, part

i: Horizontal and vertical structures. J. Atmos. Sci., 64, 3406 .

\section{List of Figures}

1 OLR power divided by background spectrum for signals (left) symmetric about the equator and (right) antisymmetric about the equator. Power spectrum is averaged over the region $15^{\circ} \mathrm{S}$ to $15^{\circ} \mathrm{N}$ and is constructed from NOAA daily OLR data running from June 1974 to September 2007. Contours begin at 1.1 and are drawn every 0.1 interval. Shallow water dispersion curves drawn are for equivalent depths of 12,25 and $50 \mathrm{~m}$, in order of increasing frequency. After Wheeler and Kiladis (1999). . . . . . . . . . . . . . . . . 46

2 Background state lower troposphere convective heating used in the control instability experiment. The background state is an idealization of the zonal mean state, with an ITCZ-like Gaussian peak over the equator and a small mean convection over the rest of the domain. $\ldots \ldots \ldots \ldots \ldots \ldots$

3 Eigenfrequencies for the linear system. Data point diameter corresponds linearly to growth rate. Stable modes omitted. Lines show theoretical dispersion curves for dry waves with equivalent depths of 40 and $60 \mathrm{~m}$ (in order of increasing frequency) . . . . . . . . . . . . . . . . .

4 Growth rates for the linear systems. Data point size encoding included for ease of cross referencing with Figure 3. The smooth curves visible to the eye correspond to the unstable wave types found in the system - Kelvin, $n=1$ Inertio-Gravity, Mixed Rossby-Gravity, and n=2 Inertio-Gravity. . . . . . . . 49 
5 Structure of the coupled Kelvin wave at planetary wavenumber 7. Shows temperature (contours) and wind anomalies (vectors) for the first and second (top and middle) baroclinic modes, as well as the reconstructed vertical temperature anomaly above the equator (bottom). . . . . . . . 50

6 Structure of the coupled Mixed Rossby-Gravity/n=0 Eastward Inertio-Gravity wave at planetary wavenumber 7 . Shows temperature (contours) and wind anomalies (vectors) for the first and second (top and middle) baroclinic modes, as well as the reconstructed vertical structure at $y=1000 \mathrm{~km}$ (north of the equator), approximately the peak of the MRG profile (bottom) . . . . . . .

7 Structure of the coupled $n=1$ Eastward Inertio-Gravity wave at planetary wavenumber 7. Shows temperature (contours) and wind anomalies (vectors) for the first and second (top and middle) baroclinic modes, as well as the reconstructed vertical temperature anomaly above the equator (bottom). . . 52

8 Structure of the coupled Westward Inertio-Gravity wave at planetary wavenumber $7(k=-7)$. Shows temperature (contours) and wind anomalies (vectors) for the first and second (top and middle) baroclinic modes, as well as the reconstructed vertical temperature anomaly above the equator (bottom). . . 53

$9 \quad$ Background state lower troposphere convection used in double ITCZ experiment. The background state is an idealization with a pair of ITCZ-like Gaussian peaks centered $1000 \mathrm{~km}$ north and south of the equator and a small mean convection over the rest of the domain. $\ldots \ldots \ldots \ldots \ldots \ldots \ldots$

10 As Figure 3, except for a background state with a double ITCZ, located near the MRG meridional structure peaks, shown in Figure 8. . . . . . . . 55
11 As Figure 3, except for a background state with a very wide ITCZ (half width $5000 \mathrm{~km})$, centered on the equator. . . . . . . . . . . . . . 56

12 Damping Sensitivity studies. As Figure 2, except for modification of the dapming paramters as follows: a) $\epsilon \rightarrow 1.1 \times \epsilon$, b) $\epsilon \rightarrow 0.9 \times \epsilon$, c) $\tau_{L} \rightarrow 1.1 \times \tau_{L}$, and d) $\tau_{L} \rightarrow 0.9 \times \tau_{L}$. Peak growth rates for each case are: a) $7 \times 10^{-2}$ day $^{-1}$, b) $9 \times 10^{-2}$ day $^{-1}$, c) $7 \times 10^{-2}$ day $^{-1}$, d) $9 \times 10^{-2}$ day $^{-1} \ldots \ldots \ldots \ldots 7$

13 As Figure 3, except for the simplified system described in Section 4 and with the dispersion curves for $h_{e q}=80 \mathrm{~m}$ also plotted. All modes with meridional index (n) greater than one are deliberately removed, despite existing within

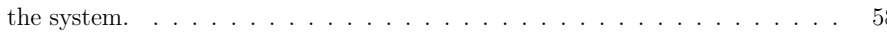

14 Analytic growth rate for the waves in the simplified limiting case, relative to the Kelvin wave growth rate (lines) and numerical growth rates (points), as described in the legend. . . . . . . . . . . . . . 59

15 Analytic divergence (top) and vorticity (bottom) fields for dry Equatorial Rossby waves with $\lambda=25 \times 10^{3} \mathrm{~km}$ (left) and $\lambda=1.1 \times 10^{3} \mathrm{~km}$ (right). It is important to note that the $\mathrm{x}$-axes of the two columns have very different scales, as demanded by the very different wavelengths depicted. Color scale is in arbitrary units. . . . . . . . . . . . . . . . . 60 

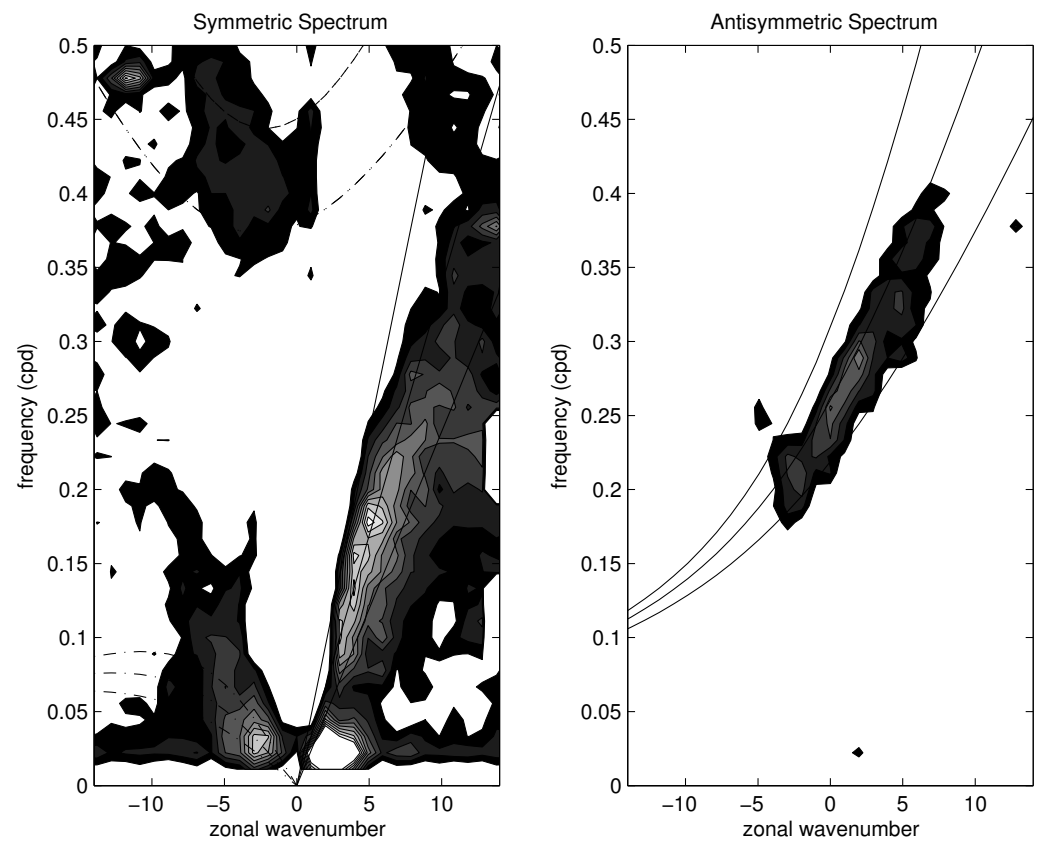

FIG. 1. OLR power divided by background spectrum for signals (left) symmetric about the

equator and (right) antisymmetric about the equator. Power spectrum is averaged over the

region $15^{\circ} \mathrm{S}$ to $15^{\circ} \mathrm{N}$ and is constructed from NOAA daily OLR data running from June 1974

to September 2007. Contours begin at 1.1 and are drawn every 0.1 interval. Shallow water

dispersion curves drawn are for equivalent depths of 12,25 and $50 \mathrm{~m}$, in order of increasing

frequency. After Wheeler and Kiladis (1999).

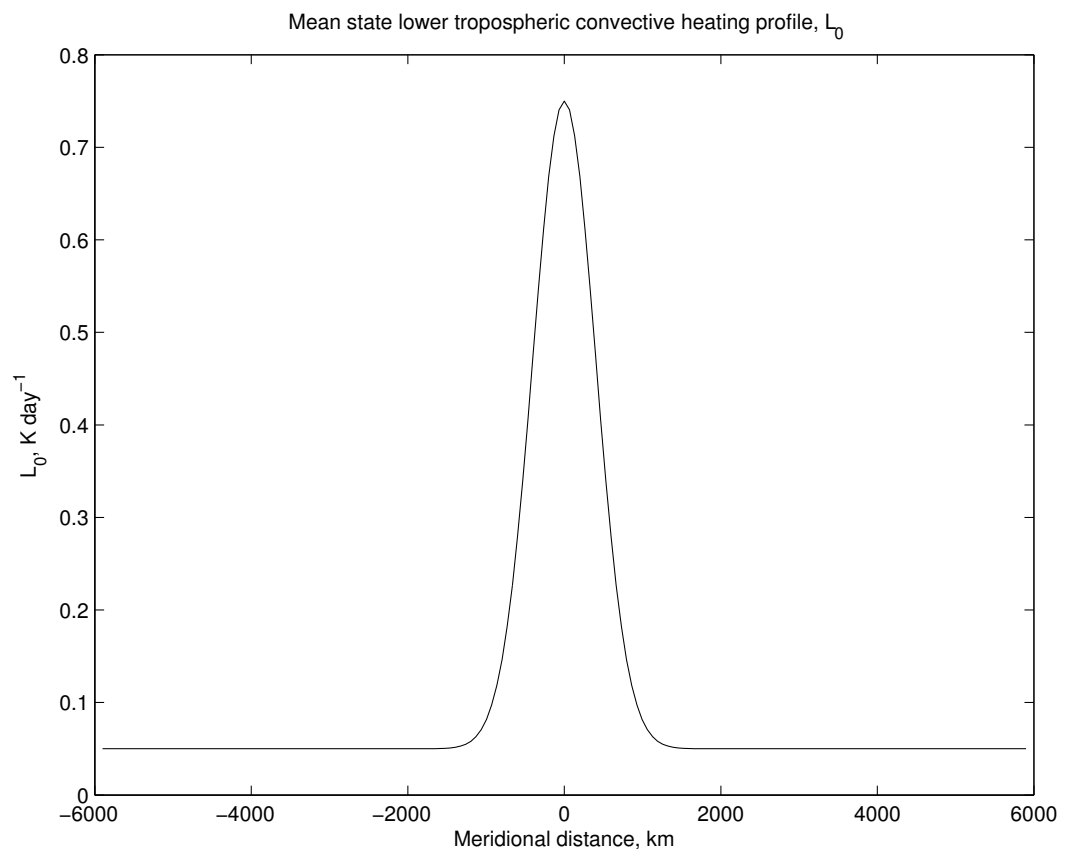

FIG. 2. Background state lower troposphere convective heating used in the control instability experiment. The background state is an idealization of the zonal mean state, with an ITCZlike Gaussian peak over the equator and a small mean convection over the rest of the domain. 


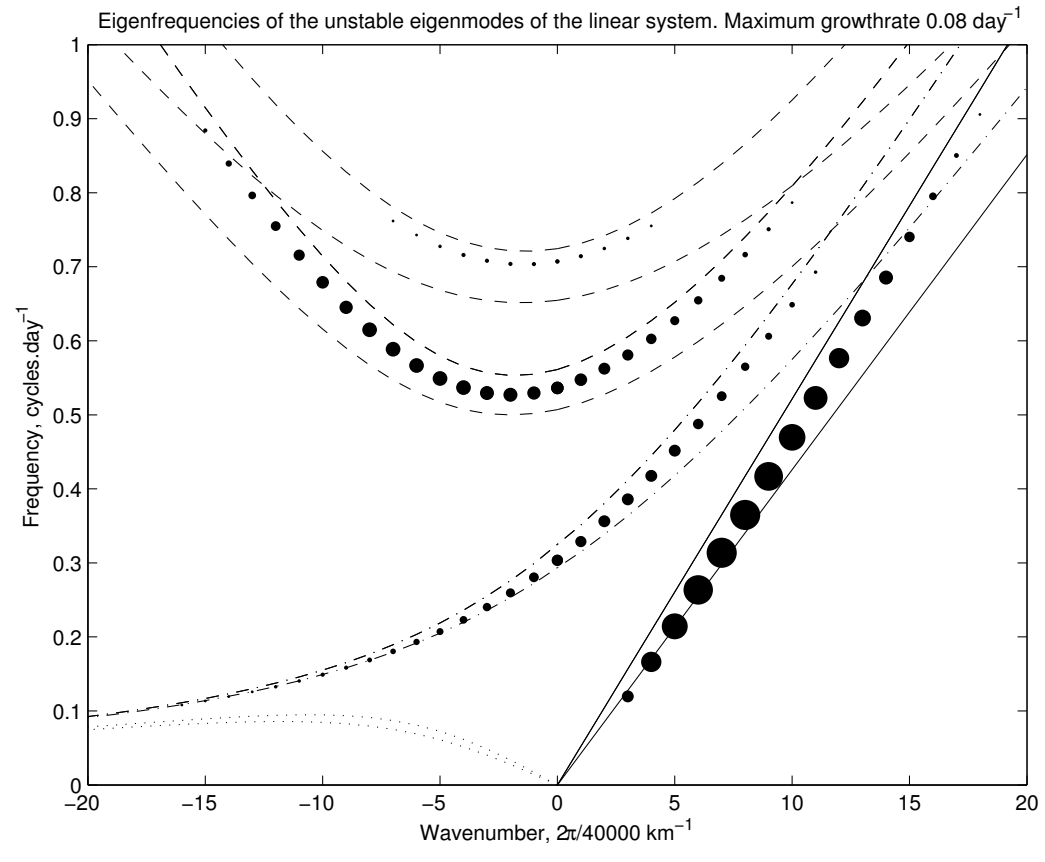

FIG. 3. Eigenfrequencies for the linear system. Data point diameter corresponds linearly to growth rate. Stable modes omitted. Lines show theoretical dispersion curves for dry waves with equivalent depths of 40 and $60 \mathrm{~m}$ (in order of increasing frequency).

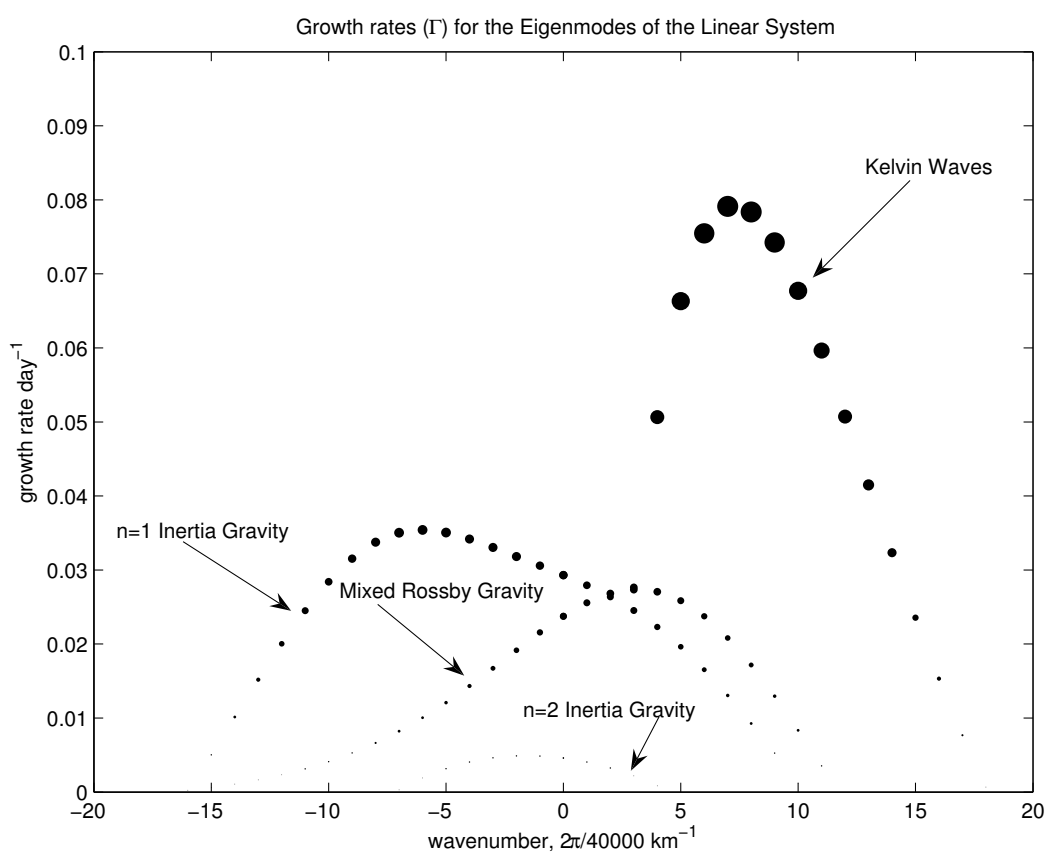

Fig. 4. Growth rates for the linear systems. Data point size encoding included for ease of cross referencing with Figure 3. The smooth curves visible to the eye correspond to the unstable wave types found in the system - Kelvin, n=1 Inertio-Gravity, Mixed RossbyGravity, and n=2 Inertio-Gravity 


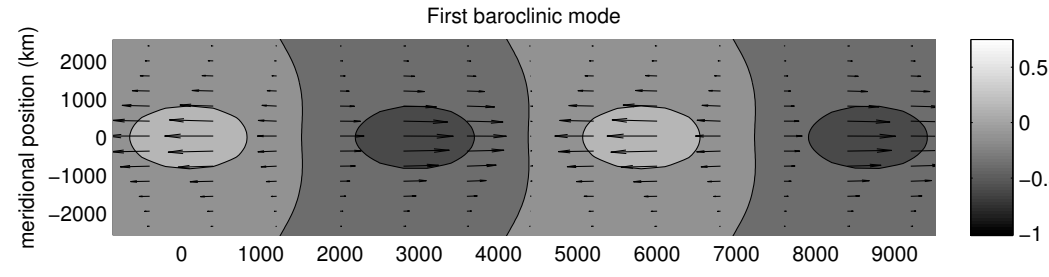
Second baroclinic mode

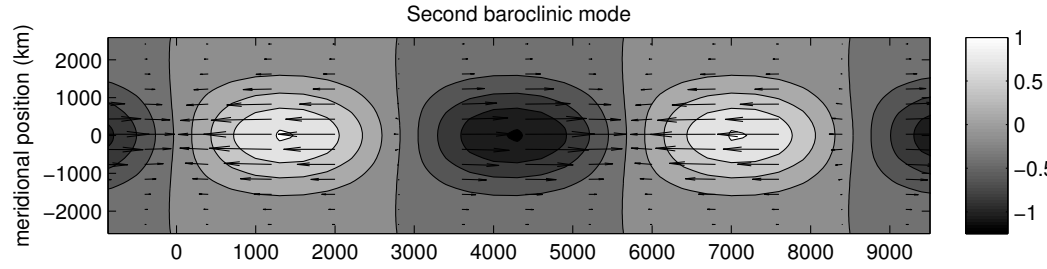

$\begin{array}{ccccccc}0 & 1000 & 2000 & 3000 & 4000 & 5000 & 6000 \\ & \text { Equatorial Vertical Temperature Structure }\end{array}$

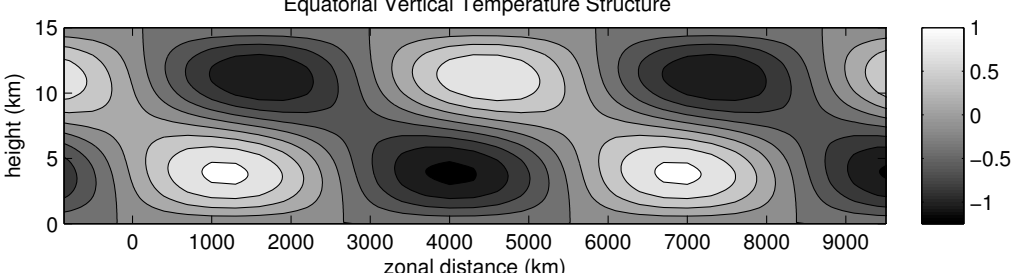

zonal distance $(\mathrm{km})$

FIG. 5. Structure of the coupled Kelvin wave at planetary wavenumber 7 . Shows temperature (contours) and wind anomalies (vectors) for the first and second (top and middle) baroclinic modes, as well as the reconstructed vertical temperature anomaly above the equator (bottom)

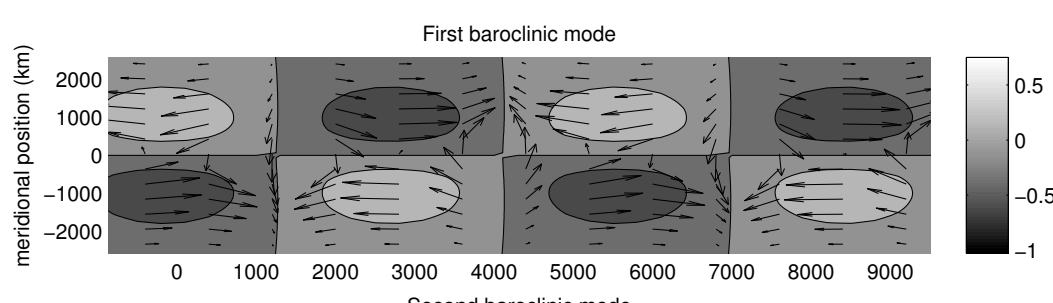
Second baroclinic mode

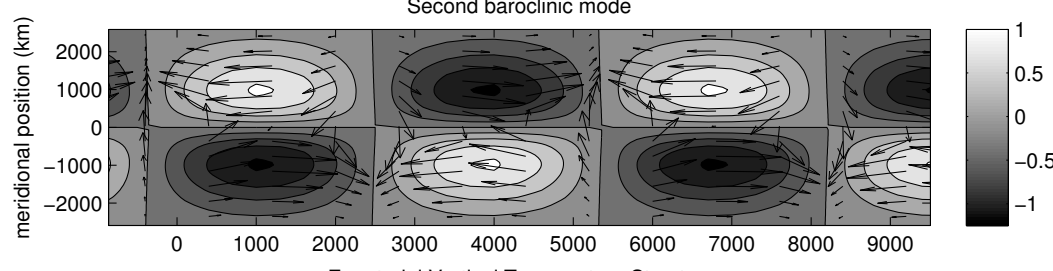

$1000 \quad 2000$ Equatorial Vertical Temperature Structure

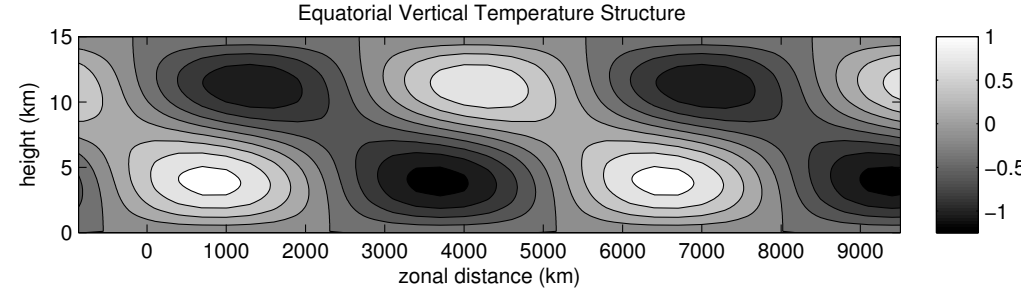

FIG. 6. Structure of the coupled Mixed Rossby-Gravity/n=0 Eastward Inertio-Gravity wave at planetary wavenumber 7 . Shows temperature (contours) and wind anomalies (vectors) for the first and second (top and middle) baroclinic modes, as well as the reconstructed vertical structure at $\mathrm{y}=1000 \mathrm{~km}$ (north of the equator), approximately the peak of the MRG profile (bottom) 


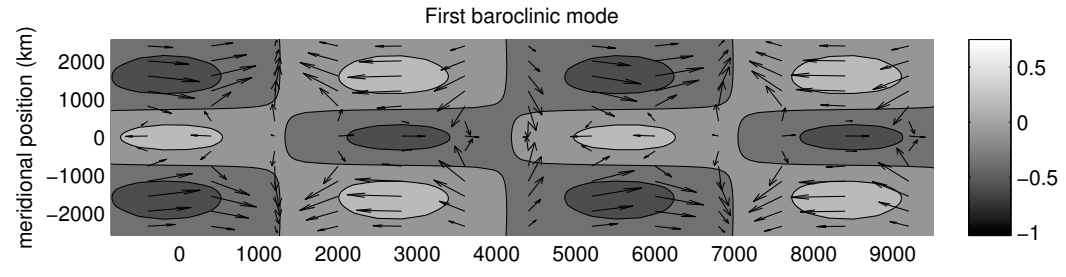

$\begin{array}{lllll}0 & 1000 \quad 2000 & 3000 \quad 4000 \quad 5000 \\ & \text { Second baroclinic mode }\end{array}$

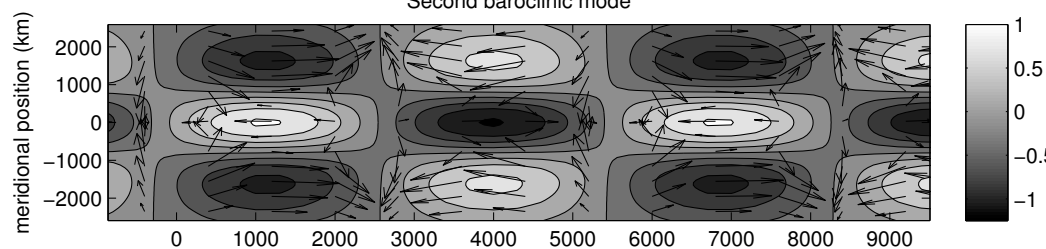

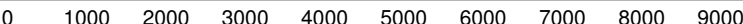
Equatorial Vertical Temperature Structure

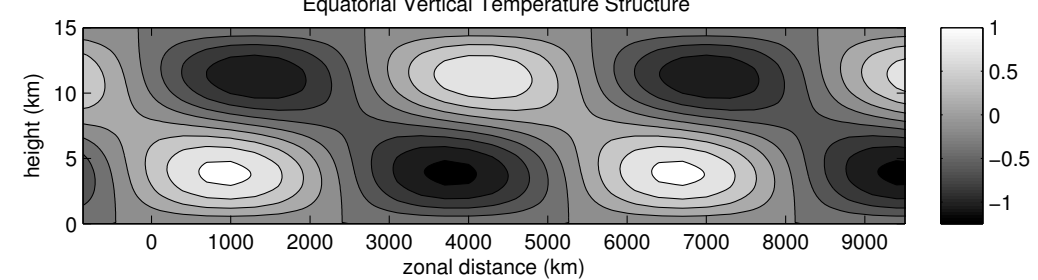

FIG. 7. Structure of the coupled n=1 Eastward Inertio-Gravity wave at planetary wavenumber 7 . Shows temperature (contours) and wind anomalies (vectors) for the first and second (top and middle) baroclinic modes, as well as the reconstructed vertical temperature anomaly above the equator (bottom)
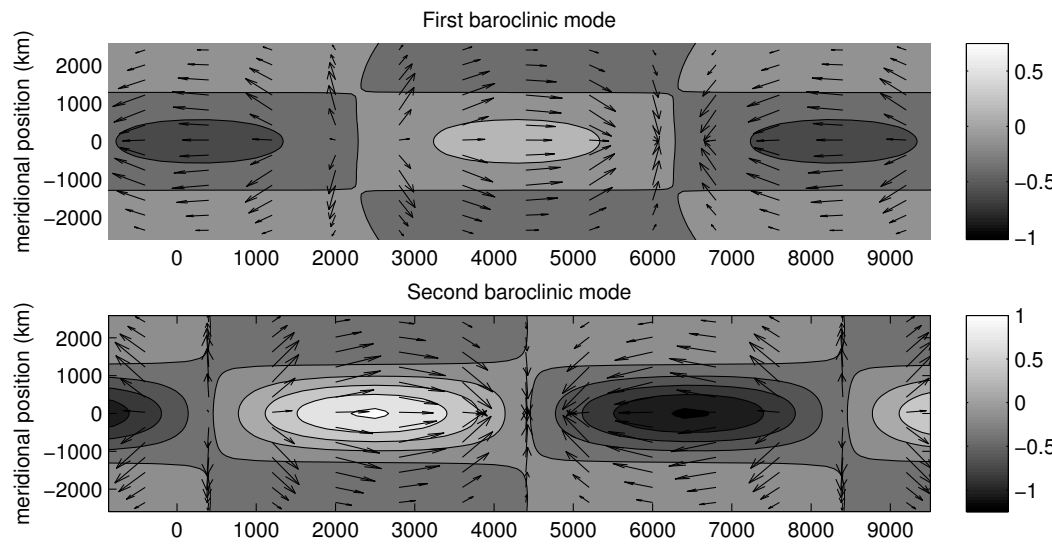

Equatorial Vertical Temperature Structure

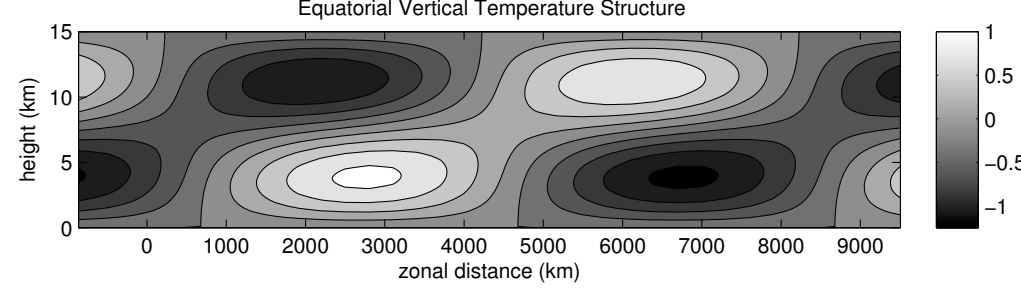

FIG. 8. Structure of the coupled Westward Inertio-Gravity wave at planetary wavenumber $7(k=-7)$. Shows temperature (contours) and wind anomalies (vectors) for the first and second (top and middle) baroclinic modes, as well as the reconstructed vertical temperature anomaly above the equator (bottom) 


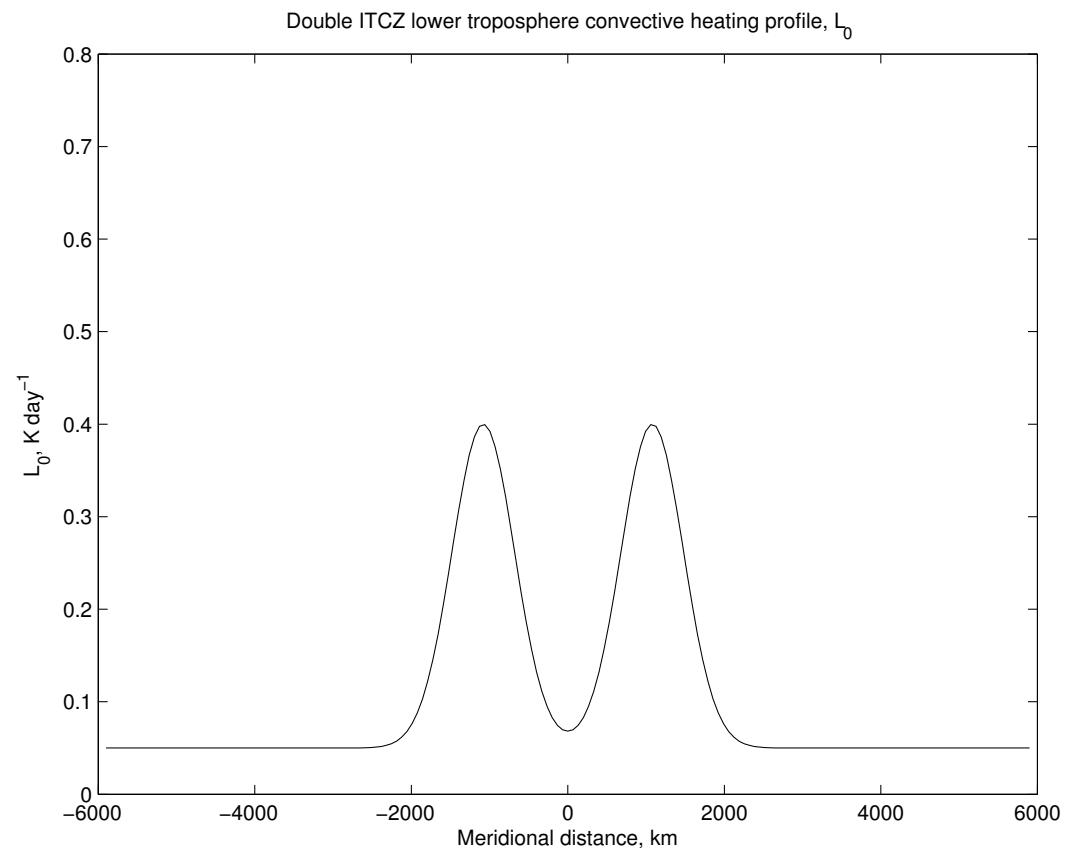

FIG. 9. Background state lower troposphere convection used in double ITCZ experiment.

The background state is an idealization with a pair of ITCZ-like Gaussian peaks centered $1000 \mathrm{~km}$ north and south of the equator and a small mean convection over the rest of the domain.

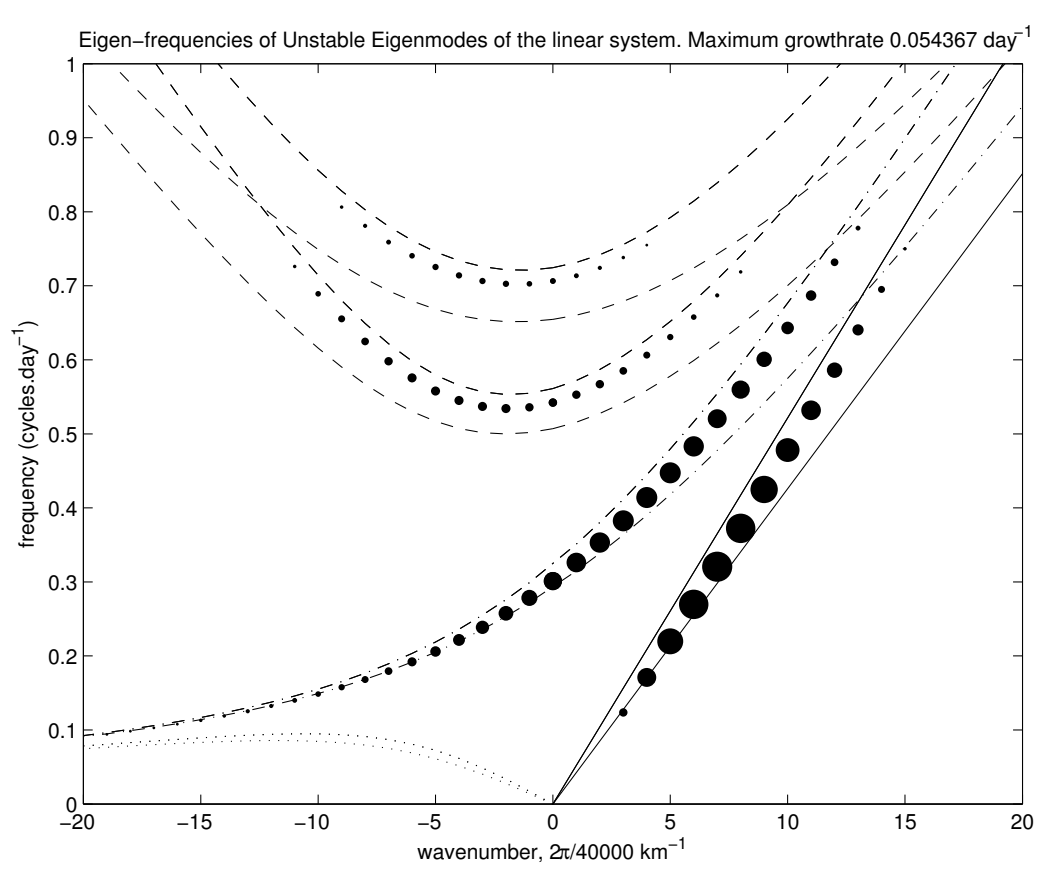

FIG. 10. As Figure 3, except for a background state with a double ITCZ, located near the MRG meridional structure peaks, shown in Figure 8. 


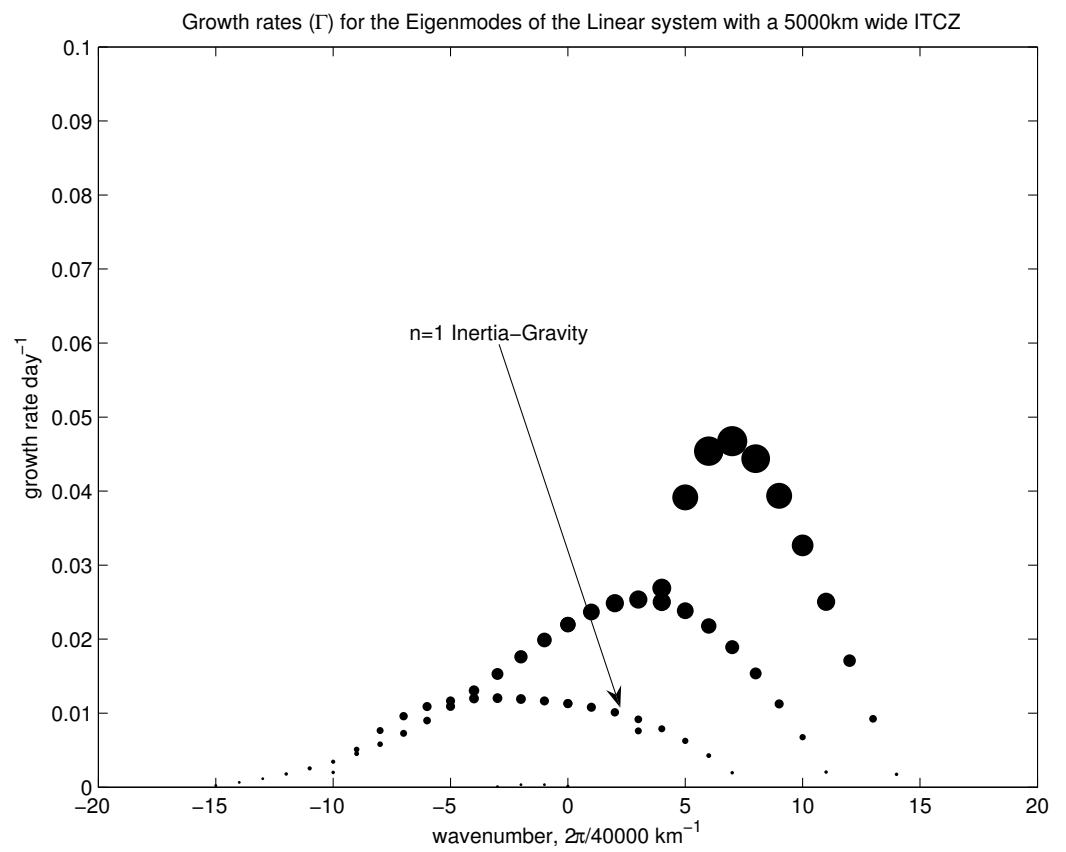

FIG. 11. As Figure 3, except for a background state with a very wide ITCZ (half width $5000 \mathrm{~km})$, centered on the equator.
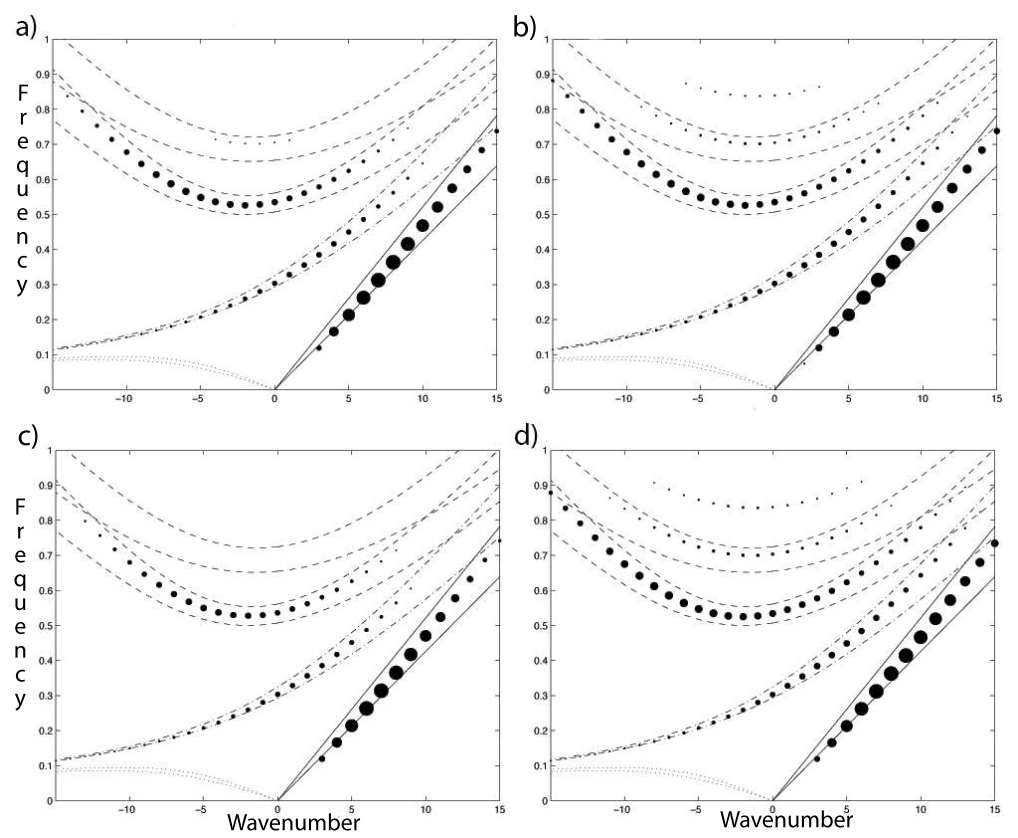

FIg. 12. Damping Sensitivity studies. As Figure 2, except for modification of the dapming paramters as follows: a) $\epsilon \rightarrow 1.1 \times \epsilon$, b) $\epsilon \rightarrow 0.9 \times \epsilon$, c) $\tau_{L} \rightarrow 1.1 \times \tau_{L}$, and d) $\tau_{L} \rightarrow 0.9 \times \tau_{L}$. Peak growth rates for each case are: a) $7 \times 10^{-2} \mathrm{day}^{-1}$, b) $9 \times 10^{-2} \mathrm{day}^{-1}$, c) $7 \times 10^{-2} \mathrm{day}^{-1}$, d) $9 \times 10^{-2}$ day $^{-1}$ 


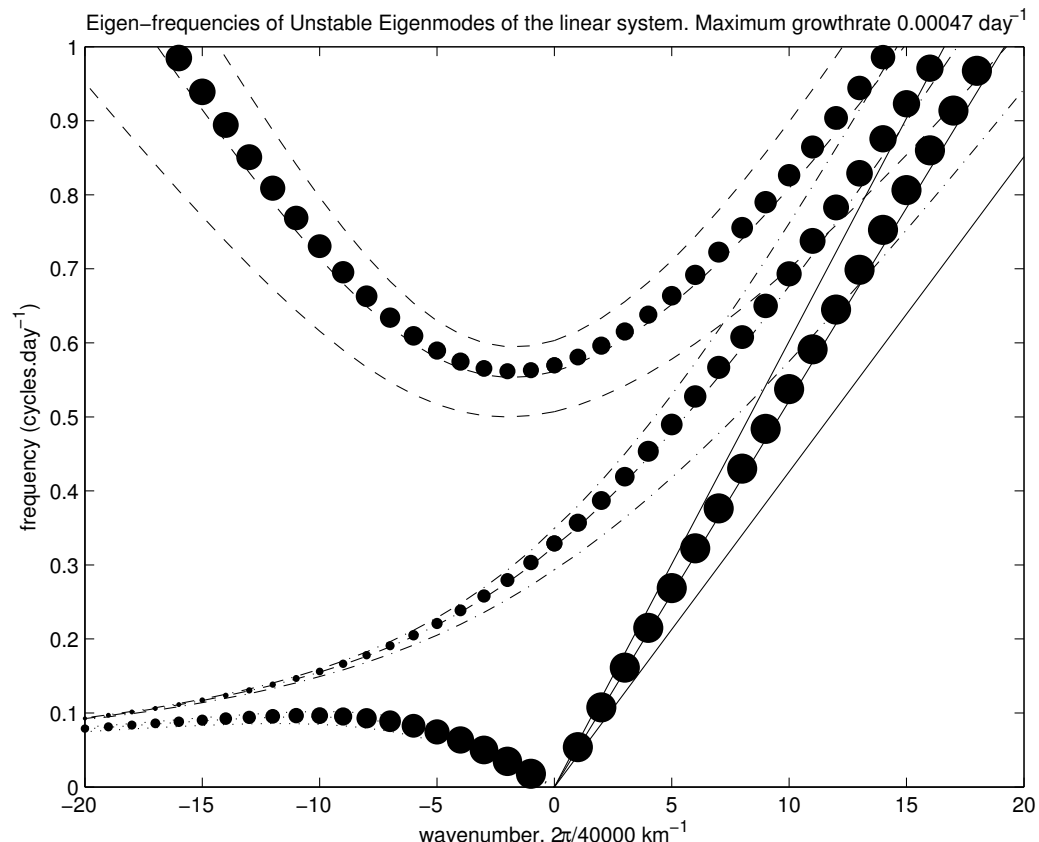

FIG. 13. As Figure 3, except for the simplified system described in Section 4 and with the dispersion curves for $h_{e q}=80 \mathrm{~m}$ also plotted. All modes with meridional index (n) greater than one are deliberately removed, despite existing within the system.

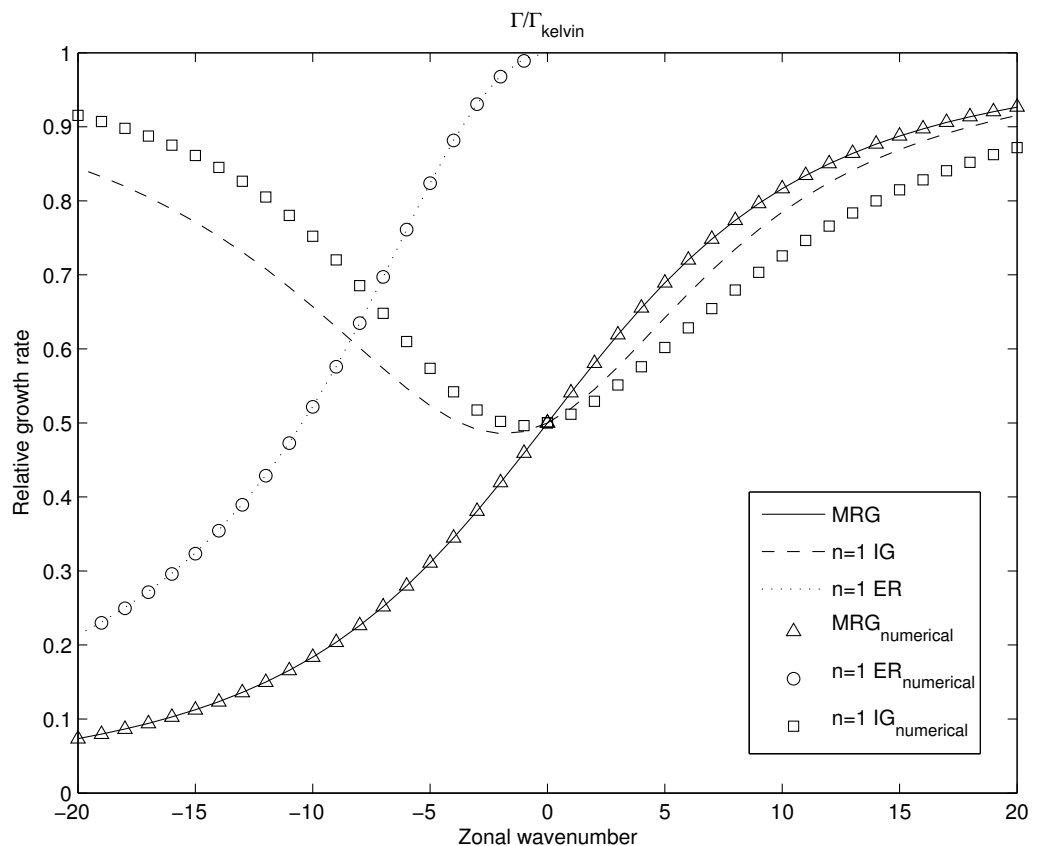

FIG. 14. Analytic growth rate for the waves in the simplified limiting case, relative to the Kelvin wave growth rate (lines) and numerical growth rates (points), as described in the legend. 


\section{List of Tables}
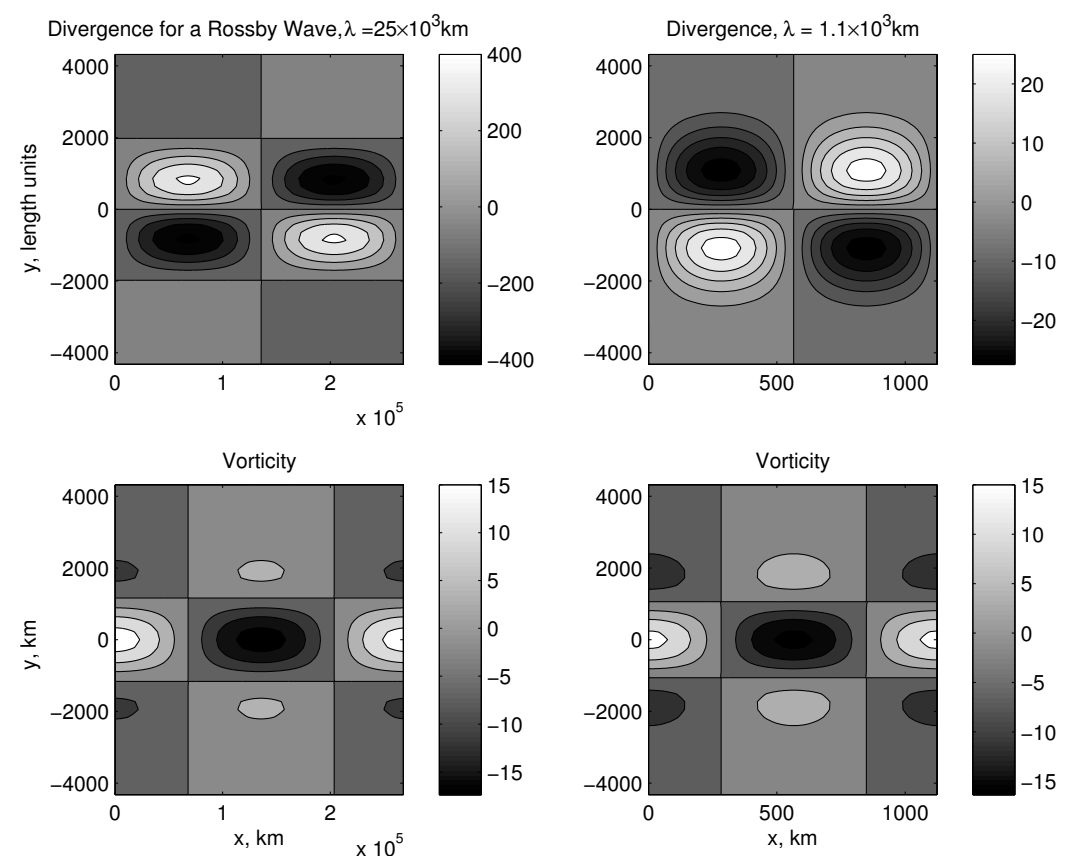

FIG. 15. Analytic divergence (top) and vorticity (bottom) fields for dry Equatorial Rossby waves with $\lambda=25 \times 10^{3} \mathrm{~km}$ (left) and $\lambda=1.1 \times 10^{3} \mathrm{~km}$ (right). It is important to note that the $\mathrm{x}$-axes of the two columns have very different scales, as demanded by the very different wavelengths depicted. Color scale is in arbitrary units. 
TABLE 1. Parameter values as used in the control cases discussed in section 3.

\begin{tabular}{|c|c|c|}
\hline Symbol & Normative Values & Description \\
\hline$b_{1}, b_{2}$ & $1.0,2.0$ & $\begin{array}{l}\text { Tendency for reduction in boundary layer moist } \\
\text { static energy per unit heating } J_{1} \text { and } J_{2}\end{array}$ \\
\hline$a_{1}, a_{2}$ & $1.4 \mathrm{~K}, 0.0 \mathrm{~K}$ & $\begin{array}{l}\text { Increase in q tendency per unit vertical velocity } \\
\left(\partial_{x} u+\partial_{y} v\right) \text { by advection }\end{array}$ \\
\hline$d_{1}, d_{2}$ & $1.1,-1.0$ & Decrease in q tendency per unit heating $J_{1}$ and $J_{2}$ \\
\hline$r_{0}$ & 1.0 & Background mean U/L ratio \\
\hline$r_{q}$ & $1.0 \mathrm{~K}^{-1}$ & Linear dependence of U/L ratio on moisture deficit \\
\hline $\mathrm{F}$ & 4 & $\begin{array}{l}\text { Ratio between moist static energy and temperature } \\
\text { in the lower tropospheric "non-entraining" convection region }\end{array}$ \\
\hline$\gamma$ & 0.5 & $\begin{array}{l}\text { Relative contribution of the first mode temperatures } \\
\text { to the lower tropospheric temperature anomaly }\end{array}$ \\
\hline$\tau_{L}$ & 2.0 hours & $\begin{array}{l}\text { Adjustment time to approach QE over the lower } \\
\text { troposphere }\end{array}$ \\
\hline$c_{1}, c_{2}$ & $(50.0,25.0) \mathrm{ms}^{-1}$ & $\begin{array}{l}\text { Dry gravity wave speeds for the first and second } \\
\text { modes }\end{array}$ \\
\hline$\epsilon$ & 0.15 day $^{-1}$ & Temperature anomaly damping coefficient \\
\hline$\sigma_{L}$ & $5.0^{\circ}$ & $\begin{array}{l}\text { Meridional width of } L_{0} \text { profile, representing } \\
\text { the ITCZ structure }\end{array}$ \\
\hline
\end{tabular}

